\title{
Prevalence and incidence of work-related musculoskeletal disorders in secondary industries of 21st century Europe: a systematic review and meta-analysis
}

Renée Govaerts ${ }^{1,2,3}$ (D) Bruno Tassignon ${ }^{2} \mathbb{D}$, Jo Ghillebert ${ }^{1,2,3}$ (D) Ben Serrien ${ }^{4} \mathbb{D}$, Sander De Bock ${ }^{1,2,3}$ (D), Toon Ampe ${ }^{2}$, llias El Makrini ${ }^{1,5}$ (D) Bram Vanderborght ${ }^{1,6}$ (D) Romain Meeusen ${ }^{1,2,3}$ (D) and Kevin De Pauw ${ }^{1,2,3^{*}}$ (D)

\begin{abstract}
Objective: Over the course of the twenty-first century, work-related musculoskeletal disorders are still persisting among blue collar workers. At present, no epidemiological overview exists. Therefore, a systematic review and meta-analysis was performed on the epidemiology of work-related musculoskeletal disorders (WMSD) within Europe's secondary industries.
\end{abstract}

Methods: Five databases were screened, yielding 34 studies for the qualitative analysis and 17 for the quantitative analysis. Twelve subgroups of WMSDs were obtained for the meta-analysis by means of predefined inclusion criteria: back (overall), upper back, lower back, neck, shoulder, neck/shoulder, elbow, wrist/hand, leg (overall), hip, knee, and ankle/feet.

Results: The most prevalent WMSDs were located at the back (overall), shoulder/neck, neck, shoulder, lower back and wrist WMSDs with mean 12-month prevalence values of 60, 54, 51, 50, 47, and 42\%, respectively. The food industry was in the majority of subgroups the most prominent researched sector and was frequently associated with high prevalence values of WMSDs. Incidence ratios of upper limb WMSDs ranged between 0.04 and 0.26. Incidence ratios could not be calculated for other anatomical regions due to the lack of sufficient articles.

Conclusion: WMSDs are still highly present among blue collar workers. Relatively high prevalence values and low incidence ratios indicate a limited onset of WMSDs with however long-term complaints.

Keywords: Epidemiology, Occupational health, Ergonomics, Musculoskeletal system, Injury, Prevention

\footnotetext{
* Correspondence: kevin.de.pauw@vub.be

${ }^{1}$ BruBotics, Vrije Universiteit Brussel, Brussels, Belgium

${ }^{2}$ Human Physiology and Sports Physiotherapy Research Group, Vrije

Universiteit Brussel, Brussels, Belgium

Full list of author information is available at the end of the article
}

C C The Author(s). 2021 Open Access This article is licensed under a Creative Commons Attribution 4.0 International License, which permits use, sharing, adaptation, distribution and reproduction in any medium or format, as long as you give appropriate credit to the original author(s) and the source, provide a link to the Creative Commons licence, and indicate if changes were made. The images or other third party material in this article are included in the article's Creative Commons licence, unless indicated otherwise in a credit line to the material. If material is not included in the article's Creative Commons licence and your intended use is not permitted by statutory regulation or exceeds the permitted use, you will need to obtain permission directly from the copyright holder. To view a copy of this licence, visit http://creativecommons.org/licenses/by/4.0/ The Creative Commons Public Domain Dedication waiver (http://creativecommons.org/publicdomain/zero/1.0/) applies to the data made available in this article, unless otherwise stated in a credit line to the data. 


\section{Introduction}

Work-related musculoskeletal disorders (WMSDs) are impairments of the musculoskeletal system, primarily caused by the performance of work tasks and the direct environment in which work is carried out [1]. Secondary industries, known for converting raw materials into products for the consumer, comprise several risk factors that contribute to the development of WMSDs [2]. Repetitive movements, awkward postures as well as continuous and excessive use of force might overload the musculoskeletal system, enhancing the risk of developing WMSDs [3]. Furthermore, psychosocial risk factors such as job related stress, lack of support from colleagues or managers, high mental workload and lack of recognition for the work done are supplementary addons in triggering the development of WMSDs in this sector [4].

The consequences of WMSDs impact both social and individual level, and result in an extensive and varied burden of costs [5]. In European industries, work absenteeism is reported in more than $50 \%$ of employees affected by WMSDs, which is significantly higher than in workers infected by the influenza virus (10-12\%) $[1,6$, 7]. Employees suffering from WMSDs are also absent from work for a longer period of time compared to workers with other health problems [1]. Furthermore, WMSDs are responsible for permanent incapacity in $60 \%$ of all reported cases [5]. Not surprisingly, the financial costs of WMSDs in Europe are estimated at 240 billion euros, accounting for $2 \%$ of the gross domestic product of EU-15 [5]. In addition to the substantial socio-economic impact, the individual employee has to pay a relatively high price as well, with studies reporting a significant decreased quality of life in people suffering from musculoskeletal disorders [8, 9]. Despite these known negative consequences, a clear epidemiological overview of WMSDs in European secondary industries is missing.

Since 2000, strategies to optimize Europe's industrial activities are constantly explored to ensure recovery from economic crises and to remain a considerable competitor to other continents $[10,11]$. Numerous research fields are therefore encouraged to develop strategies for improving overall industrial work. These innovations change the familiar way of industrial work performance (e.g. robots, exoskeletons) and could therefore impact the development of WMSDs due to changed physical and psychosocial demands $[12,13]$. However, in order to objectify the impact of these industrial technologies, recent epidemiological data regarding WMSDs in the previous setting, thus without these technological advances, are necessary first.

Due to the increased risk of incurring WMSDs in secondary industries and the detrimental impact of WMSDs in general, as well as the lack of a clear epidemiological overview on WMSDs in secondary industries of twenty-first century Europe, a systematic review and meta-analysis were performed. The aim was to provide an overall insight on the prevalence and incidence of WMSDs in Europe's secondary industries during the twenty-first century.

\section{Methods}

The review and meta-analysis was developed and reported in accordance with the preferred reporting items for systematic reviews and meta-analyses [14].

\section{Search strategy}

The PubMed, Web of Science, ScienceDirect, Cochrane library and Scopus databases, were searched for eligible articles. The final search of the databases was performed on the eight of March 2021. Three authors (RG, BT, JG) developed the search strategy in accordance with the PECO framework [15] that comprised key-words related to prevalence and incidence numbers (epidemiology OR incidence OR prevalence), industrial work (industry OR industrial worker OR industrial work OR industrial workplace OR industrial task) and musculoskeletal disorders (musculoskeletal pain OR occupational injury OR injury OR musculoskeletal disorder OR musculoskeletal disease OR musculoskeletal complaint OR musculoskeletal pain OR cumulative trauma disorders). No filters were added with the exception of publication year set from 2000 to 2021 to only include articles that researched WMSDs in the twenty-first century. In addition, reference lists of studies included in this review were screened for relevant articles not provided by the initial search strategy. Detailed descriptions for each database are displayed in supplementary figure A.

\section{Eligibility criteria}

Studies were included if they (i) provided prevalence or incidence data, defined in accordance with the definitions of incidence and prevalence provided by the Centers for Disease Control and Prevention (CDC) [16], (ii) focused on manual work in secondary industry (manufacturing), (iii) included countries of the European Union (EU-28), (iv) reported WMSDs that corresponded to an anatomical region (e.g. neck, back, hip, etc.), (v) adopted an observational study design (cross-sectional, cohort, or health surveys), (vi) used validated or nonvalidated questionnaires and (vii) were published in peer-reviewed journals. Studies were excluded when they (i) did not provide an overall number of WMSDs in secondary industry, (ii) did not make a clear distinction in manual or administrative workers for reported WMSD data, (iii) failed to clearly differentiate between industry sector, (iv) presented prevalence/incidence numbers 
based on claims or hospital records and (v) were published before the year 2000 .

\section{Study selection}

Databases were searched by one author (RG) and articles were imported in the Rayyan web application for duplicate removal and screening [17]. First, articles were screened on title and abstract by one author according to above mentioned and predefined eligibility criteria. Next, remaining articles were screened on full text by two independent researchers (RG and BT). Disagreements were solved through discussion. If consensus could not be accomplished, a third researcher (JG) would take part in the process to make inclusion by majority possible. When full texts could not be found or data of interest for this meta-analysis was missing, authors would be contacted through e-mail to request full text or data.

\section{Data extraction and risk of bias}

One author (RG) extracted the following data of included full texts to answer the research question: study design, type of industry, period of measurement, response rate, demographic characteristics of included participants (age and gender), tools for examination of WMSDs, examiner (e.g. self-report or occupational physician), type of WMSDs (e.g. neck pain or shoulder pain), prevalence and incidence data per WMSD. Occupational physicians were selected as representative for the examiner variable when options between different healthcare professionals were given. Furthermore, selfreporting prevalence data took precedence over physical examination data when studies reported both. Results will be subdivided in a Prevalence section containing a qualitative and quantitative analysis, and an Incidence section limited to a quantitative analysis.

Risk of bias was assessed in accordance with Hoy et al. [18]. They used a risk of bias tool specifically developed for prevalence studies. Risk of bias was verified through ten questions that can be answered with "high risk" or "low risk". Questions one to four assess the selection and nonresponse bias (external validity), five to nine the measurement bias and ten the bias related to the analysis. When articles provided insufficient information to answer a question a "high risk"- score was assigned to that item. Risk of bias was analyzed by two independent researchers (RG and JG). Consensus was established through discussion.

\section{Quantitative synthesis}

In order to perform a meta-analysis, studies were included if they reported (i) sufficient demographic information regarding sample size and (ii) data for specific anatomical locations i.e. neck, shoulder, elbow, wrist/hand, back or leg
WMSDs. Twelve subgroups of WMSDs were formulated: neck, shoulder, shoulder/neck, elbow, wrist/hand, upper back, lower back, back (for studies that did not make a distinction between upper or lower back), hip, knee, ankle/feet, leg (for studies that did not make a distinction between hip, knee or ankle/feet). Pooling of data occurred in several stages. First, overall prevalence percentages or incidence ratios were calculated for studies reporting data related to subgroups of the investigated sample size i.e. age, gender, skill level (e.g. unskilled versus skilled), workload (e.g. low or high) or type of manual workers (e.g. welder, metal worker, other manual workers). Overall prevalence percentages were calculated using following formula where " $\mathrm{p}$ " corresponds to prevalence, " $\mathrm{n}$ " to sample size and " $n_{\text {tot" }}$ to the sum of all sample sizes: $\left[\left(p_{1}+\right.\right.$ $\left.\left.\ldots+p_{x}\right)\left(n_{1}+\ldots+n_{x}\right)\right] / n_{\text {tot }}$. Overall incidence ratios were calculated using following formula where "i" corresponds to incidence cases, " $\mathrm{n}$ " to sample size and " $\mathrm{y}$ " to the persons-years at risk: $\left(\mathrm{i}_{1}+\ldots+\mathrm{i}_{\mathrm{x}}\right) /\left[\left(\mathrm{n}_{1}+\ldots+\mathrm{n}_{\mathrm{x}}\right)\left(\mathrm{y}_{1}+\ldots+\right.\right.$ $\left.\mathrm{y}_{\mathrm{x}}\right)$ ]. In order to minimize heterogeneity, no overall prevalence percentages or incidence ratios were calculated for studies that included prevalence periods (e.g. 12-month or 7 -day prevalence). Second, standard errors were calculated for each prevalence rate or incidence ratio using following formula with " $\mathrm{p}$ " corresponding to either prevalence rate or incidence ratio and " $n$ " to the sample size: sqrt [p (1-p) / n)]. All calculations were performed in Microsoft Excel (version 2002). Third, mean prevalence and incidence values with associated 95\% confidence intervals and heterogeneity ( $\mathrm{I}^{2}$ statistics) per WMSD-subgroup were calculated through the random-effects model of the $\mathrm{R}$ software program (version R-4.0.2). $\mathrm{I}^{2}$ statistics displays the variation across included studies that is due to heterogeneity rather than chance [19].

\section{Results \\ Study collection}

A total of 4371 articles were retrieved. After removing duplicates, 3509 articles were subsequently screened by title and abstract. The remaining 88 articles were evaluated on full text as well as six additional articles, obtained from consulting the references of included articles. A total of 35 studies were included in the qualitative analysis of which 24 authors [20-43] discussed prevalence of WMSDs, seven authors [44-50] researched incidence of WMSDs and four authors [5154] reported both incidence and prevalence rates. Figure 1 displays the results of the screening process in more detail. Due to the limited amount of incidence reports, a meta-analysis could only be performed for studies that reported prevalence data and complied with the predetermined eligibility criteria for quantitative analysis $(n=17)$. 
Identification of studies via databases and registers

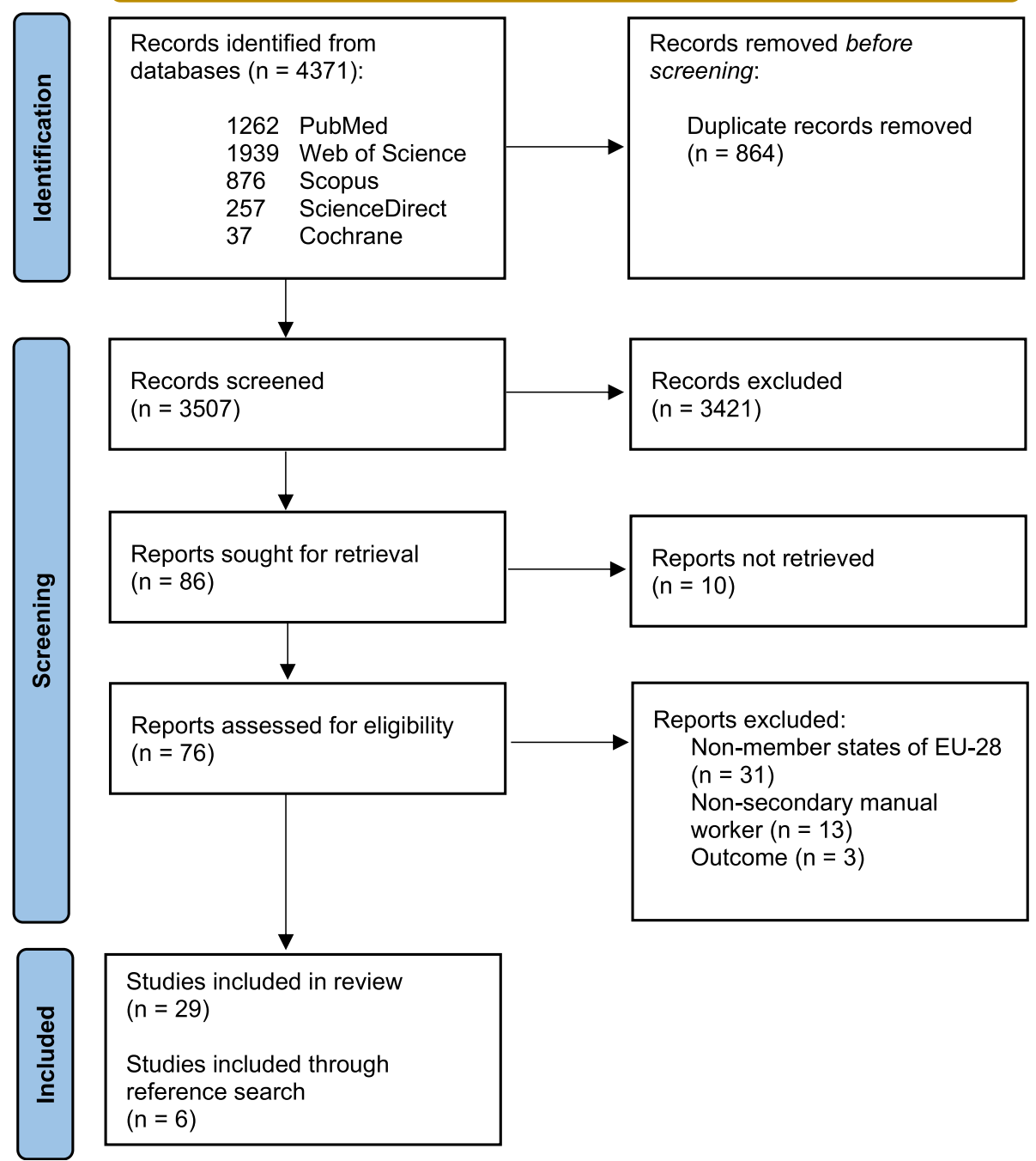

Fig. 1 Screening process

\section{Risk of bias assessment}

Risk of bias assessment resulted in 12 low risk scored studies [20, 22, 25, 31, 32, 34, 39-42, 53, 54], 20 studies with moderate risk scores [21, 23, 24, 26, 28-30, 33, 35$38,43,45-49,51,52]$ and 3 studies that received high risk scores $[27,44,50]$.

\section{Prevalence}

\section{Qualitative analysis}

The majority of studies was published between 2000 and 2010 [20, 22-25, 27, 28, 30-33, 36-40, 51, 52, 54] and most included articles were cross-sectionally designed [20-22, 2426, 29, 31, 32, 34-43, 51, 53]. Thirteen countries and seven different industries were obtained. Further, the majority of studies reported response rates greater than 50\% [20-23, 25, $27,30-34,37,38,40-42,53,54]$ and selected a sample size that ranged between 100 and 500 participants [20, 27, 28, 31-34, 36-38, 40-43, 52-54]. Assessments relying on selfreport of the workers were the most prominent tool to evaluate WMSDs [20-24, 30, 31, 35, 37, 38, 40-43]. The Nordic musculoskeletal questionnaire or a modified version was the most utilized questionnaire to identify WMSDs [22, 26, 29, 31, 34, 35, 37, 39, 42, 43, 51] (Table 1).

Although the preponderance of studies reported prevalence data over a 12-month period [20-24, 28, $31,35,40,42,43,51,54]$ or discussed point prevalence data $[25,27,32,33,52,53]$, prevalence periods varied for other included articles [26, 30, 37-39, 41]. WMSDs were mostly reported in anatomical regions of the neck, shoulder, elbow, hand/wrist, back and legs. Consequently, these anatomical regions are included in the quantitative analysis (3.3.2 Quantitative 
Table 1 Qualitative overview of prevalence of WMSDs in secondary industries of Europe

\begin{tabular}{|c|c|c|c|c|c|}
\hline $\begin{array}{l}\text { Author, year, } \\
\text { study design, } \\
\text { industry \& country }\end{array}$ & $\begin{array}{l}\text { Period of } \\
\text { measurement }\end{array}$ & $\begin{array}{l}\text { Demographic } \\
\text { characteristics }\end{array}$ & $\begin{array}{l}\text { Examination } \\
\text { method \& } \\
\text { response rate }\end{array}$ & Tools for examination & $\begin{array}{l}\text { WMSD \& } \\
\text { Prevalence }\end{array}$ \\
\hline $\begin{array}{l}\text { Aasmoe et al. } 2008 \text { [20] } \\
\text { Cross-sectional } \\
\text { Seafood industry - Norway }\end{array}$ & $\begin{array}{l}\text { December } \\
2000 \\
\text { Prevalence } \\
\text { Period: } \\
12 \text { months }\end{array}$ & $\begin{array}{l}T=744 \\
F=85(21 \%) ; \\
<30 \text { years } \\
F=97(24 \%) ; \\
30-39 \text { years } \\
F=109(27 \%) ; \\
40-49 \text { years } \\
F=113(28 \%) ; \\
>50 \text { years } \\
M=119(35 \%) ; \\
<30 \text { years } \\
M=85(25 \%) ; \\
30-39 \text { years } \\
M=71(21 \%) ; \\
40-49 \text { years } \\
M=64(19 \%) ; \\
>50 \text { years }\end{array}$ & $\begin{array}{l}\text { Self-report } \\
50 \% \text { of the } \\
\text { administrative and } \\
\text { production } \\
\text { workers }\end{array}$ & $\begin{array}{l}\text { Self-Administered Questionnaire } \\
\text { (anonymous): "Have you had any of } \\
\text { these symptoms during the last } 12 \\
\text { months?" }\end{array}$ & 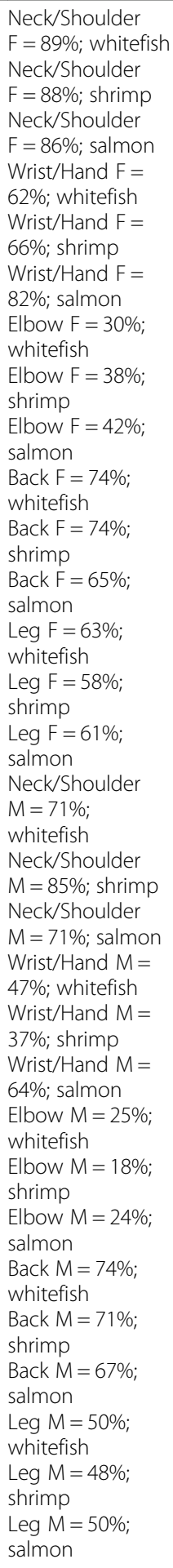 \\
\hline $\begin{array}{l}\text { Afonso et al. } 2014 \text { [21] } \\
\text { Cross-sectional } \\
\text { Footwear industry - Portugal }\end{array}$ & $\begin{array}{l}\text { January } 2013 \\
\text { Prevalence } \\
\text { Period: } \\
12 \text { months }\end{array}$ & $\begin{array}{l}F=66 ; 22-55 \\
\text { years }\end{array}$ & $\begin{array}{l}\text { Self-report } \\
52.3 \%\end{array}$ & $\begin{array}{l}\text { Questionnaire based on } 3 \text { validated } \\
\text { questionnaires: } \\
\text { 1) Nordic Musculoskeletal } \\
\text { Questionnaire translated in } \\
\text { Portuguese }\end{array}$ & $\begin{array}{l}\text { Neck T }=32 \% \\
(n=21) \\
\text { Shoulder T }=23 \% \\
(n=15) \\
\text { Elbow T }=21 \%\end{array}$ \\
\hline
\end{tabular}


Table 1 Qualitative overview of prevalence of WMSDs in secondary industries of Europe (Continued)

\begin{tabular}{|c|c|c|c|c|}
\hline $\begin{array}{l}\text { Author, year, } \\
\text { study design, } \\
\text { industry \& country }\end{array}$ & $\begin{array}{l}\text { Period of } \\
\text { measurement }\end{array}$ & $\begin{array}{l}\text { Demographic } \\
\text { characteristics }\end{array}$ & $\begin{array}{l}\text { Examination } \\
\text { method \& } \\
\text { response rate }\end{array}$ & Tools for examination \\
\hline & & & & $\begin{array}{l}\text { 2) Extended version of the Dutch } \\
\text { Musculoskeletal Questionnaire } \\
\text { 3) Medium size version of the } \\
\text { Copenhagen Psychosocial } \\
\text { Questionnaire }\end{array}$ \\
\hline $\begin{array}{l}\text { Alexopoulos et al. } 2006 \text { [22] } \\
\text { Cross-sectional } \\
\text { Shipyard industry - Greece }\end{array}$ & $\begin{array}{l}\text { November } \\
\text { 2003-March } \\
2004 \\
\text { Prevalence } \\
\text { Period: } \\
12 \text { months }\end{array}$ & $\begin{array}{l}\text { Blue collar } \\
\text { workers }=624 ; \\
\bar{y}=38.7 \text { years }\end{array}$ & $\begin{array}{l}\text { Self-report } \\
98.5 \% \text { of the white } \\
\text { and blue collar } \\
\text { workers }\end{array}$ & $\begin{array}{l}\text { Nordic Musculoskeletal } \\
\text { Questionnaire translated into Greek }\end{array}$ \\
\hline
\end{tabular}

WMSD \&

Prevalence

\section{$(n=14)$}

Wrist/Hand $\mathrm{T}=$

$42 \%(n=18)$

Thoracic region

$\mathrm{T}=6 \%(n=4)$

Lumbar region

$\mathrm{T}=30 \%(n=20)$

Hip/Thigh T = 9\%

$(n=6)$

Knee $T=21 \%$

$(n=14)$

Ankle/Foot $\mathrm{T}=$

$15 \%(n=10)$

Lower Back =

33\%; metal

workers

Lower Back =

39.8\%; welders

Lower Back =

37.9\%; other blue

collar workers

Shoulder/Neck =

14.6\%; metal

workers

Shoulder/Neck =

18.3\%; welders

Shoulder/Neck =

25.2\%; other blue

collar workers

Hand/Wrist =

15.3\%; metal

workers

Hand/Wrist =

10.8\%; welders

Hand/Wrist =

13.4\%; other blue collar workers

Andersen et al. 2007 [23]

Cohort

2-year period $T=141$; skilled Self-report

Prevalence workers 75\%; skilled

Period: $\quad T=1874 ; \quad$ workers baseline

12 months
Self-Administered Questionnaire for regional pain status: "How much have you been bothered by pain during the past 12 months?"
$67 \%$; unskilled

workers baseline

79\%; skilled

workers 24-month

follow-up

76\%; unskilled

workers 24-month

follow-up
Neck/Shoulder $\mathrm{T}=22 \%$; skilled workers baseline Elbow/Forearm/ Hand $\mathrm{T}=17 \%$; skilled workers baseline

Lower Back T = 17\%; skilled workers baseline Hip/Knee/Foot $\mathrm{T}=12 \%$; skilled workers baseline Neck/Shoulder $\mathrm{T}=38 \%$; unskilled workers baseline Elbow/Forearm/ Hand T $=22 \%$; unskilled workers baseline

Lower Back T= 25\%; unskilled workers baseline Hip/Knee/Foot $\mathrm{T}=22 \%$; unskilled workers baseline Neck/Shoulder $\mathrm{T}=16 \%$; skilled 
Table 1 Qualitative overview of prevalence of WMSDs in secondary industries of Europe (Continued)

Author, year, Period of Demographic Examination Tools for examinatio study design, industry \& country

\section{measurement characteristics method \&} response rate
Bang et al. 2005 [24]

Cross-sectional

Seafood industry - Norway

Bonfiglioli et al. 2006 [25]

Cross-sectional

Electronic industry - Italy

Claus et al. 2019 [26]

Cross-sectional

Chemical industry - Germany

$\begin{array}{lll}2001 & T=1588 ; m= & \begin{array}{l}\text { Self-report } \\ 49.8 \% \text { of the }\end{array} \\ \text { Prevalence } & \text { 39years } & \text { industrial and } \\ \text { Period: } & F=889(56 \%) & \begin{array}{l}\text { administrative } \\ \text { workers }\end{array}\end{array}$

Not

mentioned

Prevalence

Period:

Point

prevalence

January 2011

December

2014

Prevalence

Period:

1) 12 months

2) 7 days

\section{$\mathrm{F}=19$ \\ $\mathrm{M}=32$ \\ $\bar{y}=36.3$ years \\ Clinician and experienced \\ electrodiagnostic tester \\ $85 \%$}

\section{Self-report}

Occupational

physicians

years

$\mathrm{T}=634$

(11.4\%); 35-39

years

$\mathrm{T}=930$

(16.7\%); 40-44

years

$\mathrm{T}=1078$

(19.4\%); 45-49

years

$\mathrm{T}=1100$

(19.8\%); 50-54

years

$\mathrm{T}=664$

$(11.9 \%) ;>55$

years

$\mathrm{F}=254(4.6 \%)$

$M=5317$
Not further specified questionnaire. Question "Have you, during the last 12 months, felt pain from the neck shoulders, elbow, wrists/hands, back and legs?"

Bilateral median nerve conduction study

Physical examination
WMSD \&

Prevalence

workers 24-

month follow-up Elbow/Forearm/

Hand $\mathrm{T}=8 \%$;

skilled workers

24-month follow-

up

Lower Back T=

19\%; skilled

workers 24-

month follow-up

Hip/Knee/Foot

$\mathrm{T}=17 \%$; skilled

workers 24-

month follow-up

Neck/Shoulder

$\mathrm{T}=32 \%$; unskilled

workers 24-

month follow-up

Elbow/Forearm/

Hand $\mathrm{T}=21 \%$;

unskilled workers

24-month followup

Lower Back $\mathrm{T}=$

27\%; unskilled

workers 24-

month follow-up

Hip/Knee/Foot

$\mathrm{T}=24 \%$; unskilled

workers 24-

month follow-up

Neck/Shoulder

$\mathrm{T}=65.3 \%$

Elbow $\mathrm{T}=15.3 \%$

Wrist/Hand T $=$

39.7\%

Back $\mathrm{T}=56.1 \%$

Leg $T=39.4 \%$

Carpal Tunnel Syndrome $\mathrm{T}=$ $43 \%$

Back $\mathrm{T}=66.4 \%$;

12-month

prevalence

Back $\mathrm{T}=26.3 \%$; 7 -

day prevalence 
Table 1 Qualitative overview of prevalence of WMSDs in secondary industries of Europe (Continued)

\begin{tabular}{|c|c|c|c|c|}
\hline $\begin{array}{l}\text { Author, year, } \\
\text { study design, } \\
\text { industry \& country }\end{array}$ & $\begin{array}{l}\text { Period of } \\
\text { measurement }\end{array}$ & $\begin{array}{l}\text { Demographic } \\
\text { characteristics }\end{array}$ & $\begin{array}{l}\text { Examination } \\
\text { method \& } \\
\text { response rate }\end{array}$ & Tools for examination \\
\hline & & $\begin{array}{l}(95.4 \%) \\
\bar{y}=43.1 \text { years }\end{array}$ & & \\
\hline $\begin{array}{l}\text { De Zwart et al. } 2001 \text { [27] } \\
\text { Cross-sectional } \\
\text { Textile, food, metal, electronic, } \\
\text { and production industry - The } \\
\text { Netherlands }\end{array}$ & $\begin{array}{l}\text { 1982-1993 } \\
\text { Prevalence } \\
\text { Period: } \\
\text { Point } \\
\text { prevalence }\end{array}$ & $\begin{array}{l}\mathrm{F}=80(30.5 \%) ; \\
\text { Textile industry } \\
\mathrm{F}=182 \\
(16.0 \%) ; \text { Food } \\
\text { and beverage } \\
\text { industry } \\
\mathrm{F}=61 \text { (11.6\%); } \\
\text { Assemblers of } \\
\text { metal products } \\
\mathrm{F}=52 \text { (17.0\%); } \\
\text { Assemblers of } \\
\text { electrical } \\
\text { products } \\
\mathrm{F}=58(17.4 \%) ; \\
\text { Production } \\
\text { and related } \\
\text { workers NEC } \\
\mathrm{M}=182 \\
\text { (69.5\%); Textile } \\
\text { industry } \\
\mathrm{M}=958 \text { (84\%); } \\
\text { Food and } \\
\text { beverage } \\
\text { industry } \\
\mathrm{M}=463 \\
\text { (88.4\%); } \\
\text { Assemblers of } \\
\text { metal products } \\
\mathrm{M}=254 \\
\text { (83.0\%); } \\
\text { Assemblers of } \\
\text { electrical } \\
\text { products } \\
\mathrm{M}=276 \\
\text { ( } 82.6 \%) ; \\
\text { Production } \\
\text { and related } \\
\text { workers NEC }\end{array}$ & $\begin{array}{l}\text { Self-report } \\
\text { Regional } \\
\text { occupational } \\
\text { health service } \\
70-90 \%\end{array}$ & $\begin{array}{l}\text { Self-Administered Questionnaire } \\
\text { Physical examination }\end{array}$ \\
\hline
\end{tabular}

WMSD \&

Prevalence industry \& country

$$
(95.4 \%)
$$$$
\bar{y}=43.1 \text { years }
$$

Textile, food, metal, electronic,

Prevalence

Period:

Point

Textile industry

$F=182$

Self-report

health service

70-90\%

Assemblers of

electrical

Production

and related

$M=182$

(69.5\%); Textile

Food and

beverage

$M=254$

mers of

$M=276$

workers NEC

\section{Prevalence}

Neck $F=20.0 \%$;

Textile industry

Neck $F=23.6 \%$;

Food and

beverage industry

Neck F = 16.4\%;

Assemblers of

metal products

Neck F $=30.8 \%$;

Assemblers of

electrical products

Neck F = 5.5\%;

Production and

related workers

NEC

Shoulder $\mathrm{F}=$

12.5\%; Textile

industry

Shoulder $\mathrm{F}=$

34.1\%; Food and

beverage industry

Shoulder $\mathrm{F}=$

18.0\%; Assemblers

of metal products

Shoulder $\mathrm{F}=$

23.1\%; Assemblers

of electrical

products

Shoulder $\mathrm{F}=$

15.5\%; Production

and related

workers NEC

Elbow $F=3.8 \%$;

Textile industry

Elbow $F=3.8 \%$;

Food and

beverage industry

Elbow $F=1.6 \%$;

Assemblers of

metal products

Elbow $F=7.7 \%$;

Assemblers of

electrical products

Elbow $F=1.7 \%$;

Production and

related workers

NEC

Wrist $F=1.3 \%$;

Textile industry

Wrist $F=13.7 \%$;

Food and

beverage industry

Wrist $F=4.9 \%$;

Assemblers of

metal products

Wrist $F=11.5 \%$;

Assemblers of

electrical products

Wrist $\mathrm{F}=0 \%$;

Production and

related workers

NEC

Neck $M=13.2 \%$;

Textile industry 
Table 1 Qualitative overview of prevalence of WMSDs in secondary industries of Europe (Continued)

Author, year, Period of Demographic Examination Tools for examination

WMSD \&

Prevalence industry \& country

Neck $M=10.6 \%$;

Food and

beverage industry Neck $M=8.6 \%$;

Assemblers of metal products Neck $M=1.8 \%$; Assemblers of electrical products Neck $M=11.2 \%$

Production and related workers NEC

Shoulder $\mathrm{M}=$ 10.4\%; Textile industry Shoulder $\mathrm{M}=$ 17.2\%; Food and beverage industry Shoulder $\mathrm{M}=$ 12.5\%; Assemblers of metal products Shoulder $\mathrm{M}=$ 13.0\%; Assemblers of electrical products

Shoulder $\mathrm{M}=$ 12.7\%; Production and related workers NEC

Elbow $M=4.4 \%$; Textile industry Elbow $\mathrm{M}=4.4 \%$; Food and beverage industry Elbow $\mathrm{M}=6.5 \%$; Assemblers of metal products Elbow $M=5.5 \%$; Assemblers of electrical products Elbow $M=3.6 \%$; Production and related workers NEC

Wrist $M=5.5 \%$; Textile industry Wrist $M=4.4 \%$; Food and beverage industry Wrist $M=4.3 \%$; Assemblers of metal products Wrist $M=3.9 \%$; Assemblers of electrical products Wrist $M=5.4 \%$; Production and related workers NEC

Descatha et al. 2003 [28]

Cohort

Assembly, textile, food, and packaging industry - France
1993-1994

Prevalence

Period:

12 months

\section{$T=479 ;$}

Assembly industry

$T=262$; Textile industry

$\mathrm{T}=307$; Food
Self-Administered Questionnaire Occupational physicians

Physical examination
Medial epicondylitis $\mathrm{T}=5.2 \%$; Assembly industry $\mathrm{T}=2.7 \%$; Textile industry 
Table 1 Qualitative overview of prevalence of WMSDs in secondary industries of Europe (Continued)

\begin{tabular}{|c|c|c|c|c|c|}
\hline $\begin{array}{l}\text { Author, year, } \\
\text { study design, } \\
\text { industry \& country }\end{array}$ & $\begin{array}{l}\text { Period of } \\
\text { measurement }\end{array}$ & $\begin{array}{l}\text { Demographic } \\
\text { characteristics }\end{array}$ & $\begin{array}{l}\text { Examination } \\
\text { method \& } \\
\text { response rate }\end{array}$ & Tools for examination & $\begin{array}{l}\text { WMSD \& } \\
\text { Prevalence }\end{array}$ \\
\hline & & $\begin{array}{l}\text { industry } \\
\mathrm{T}=160 ; \\
\text { Packaging } \\
\text { industry }\end{array}$ & & & $\begin{array}{l}\mathrm{T}=5.2 \% ; \text { Food } \\
\text { industry } \\
\mathrm{T}=2.5 \% \\
\text { Packaging } \\
\text { industry }\end{array}$ \\
\hline $\begin{array}{l}\text { Fouquet et al. } 2015 \text { [29] } \\
\text { Cross-sectional } \\
\text { Agriculture and food, automotive } \\
\text { manufacturing, and energy } \\
\text { industry - France }\end{array}$ & $\begin{array}{l}\text { 2002-2005 } \\
\text { Prevalence } \\
\text { Period: } \\
7 \text { days }\end{array}$ & $\begin{array}{l}\text { Not } \\
\text { mentioned }\end{array}$ & $\begin{array}{l}\text { Occupational } \\
\text { physicians } \\
18 \% \text { of the } \\
\text { occupational } \\
\text { physicians (all } \\
\text { industries) }\end{array}$ & $\begin{array}{l}\text { Nordic Musculoskeletal } \\
\text { Questionnaire }\end{array}$ & $\begin{array}{l}\text { Thoracic spine } \\
\mathrm{F}=15.0 \% \text { ( } \mathrm{n}= \\
113) ; \text { Agricultural } \\
\text { and food industry } \\
\mathrm{F}=19.8 \% \text { ( } n= \\
116) ; \text { Automotive } \\
\text { manufacturing } \\
\text { industry } \\
\mathrm{F}=0 \%(n=0) ; \\
\text { Energy } \\
M=10.4 \% \text { ( } n= \\
182) ; \text { Agricultural } \\
\text { and food industry } \\
M=19.4 \% \text { ( } n= \\
62) ; \text { Automotive } \\
\text { manufacturing } \\
\text { industry } \\
M=8.3 \% \text { ( } n=12 \text { ); } \\
\text { Energy industry }\end{array}$ \\
\hline $\begin{array}{l}\text { Ha et al. } 2009 \text { [51] } \\
\text { Cross-sectional } \\
\text { Food and drink, garment, shoe } \\
\text { and leather, manufacture of } \\
\text { wood and wood products, } \\
\text { manufacture of pulp, paper and } \\
\text { paper products, publishing, } \\
\text { printing and reproduction of } \\
\text { recorded media, chemical, } \\
\text { manufacture of rubber and } \\
\text { plastic products, manufacture of } \\
\text { other non-metallic mineral prod- } \\
\text { ucts, manufacture of basic } \\
\text { metals, manufacture of fabricated } \\
\text { metal products, manufacture of } \\
\text { machinery and equipment not } \\
\text { elsewhere classified, manufacture } \\
\text { of electrical and optical equip- } \\
\text { ment, manufacture of motor ve- } \\
\text { hicles, manufacture of other } \\
\text { transport equipment, manufac- } \\
\text { ture of furniture and wood, re- } \\
\text { cycling industry - France }\end{array}$ & $\begin{array}{l}\text { April 2002- } \\
\text { April } 2005 \\
\text { Prevalence } \\
\text { Period: } \\
12 \text { months }\end{array}$ & $\begin{array}{l}\mathrm{F}=113 ; \text { Food } \\
\text { and drink } \\
\text { industry } \\
\mathrm{F}=12 ; \\
\text { Garment } \\
\text { industry } \\
\mathrm{F}=28 ; \text { Shoe } \\
\text { and leather } \\
\text { industry } \\
\mathrm{F}=6 ; \\
\text { Manufacture of } \\
\text { wood and } \\
\text { wood } \\
\text { products } \\
\mathrm{F}=12 ; \text { Pulp, } \\
\text { paper and } \\
\text { paper products } \\
\text { manufacturing } \\
\mathrm{F}=9 ; \\
\text { Publishing, } \\
\text { printing, } \\
\text { reproduction } \\
\text { of recorded } \\
\text { media } \\
\mathrm{F}=2 ; \text { Chemical } \\
\text { industry } \\
\mathrm{F}=45 ; \\
\text { Manufacture of } \\
\text { rubber and } \\
\text { plastic } \\
\text { products } \\
\mathrm{F}=2 ; \text { Non- } \\
\text { metallic min- } \\
\text { eral products } \\
\text { manufacturing } \\
\mathrm{F}=6 ; \\
\text { Manufacture of } \\
\text { basic metals } \\
\mathrm{F}=11 ; \\
\text { Manufacture of } \\
\text { fabricated }\end{array}$ & $\begin{array}{l}\text { Neurologists } \\
\text { Occupational } \\
\text { physicians } \\
17.4 \% \text { of the } \\
\text { occupational } \\
\text { physicians }\end{array}$ & $\begin{array}{l}\text { Nordic Musculoskeletal } \\
\text { Questionnaire } \\
\text { Physical examination according the } \\
\text { "criteria document" (if symptoms } \\
\text { had occurred during the last } 12 \\
\text { months) }\end{array}$ & $\begin{array}{l}\text { Upper limb } \\
\text { musculoskeletal } \\
\text { disorder } \\
\mathrm{F}=13.3 \% \text {; Food } \\
\text { and drink industry } \\
\mathrm{F}=33.3 \% ; \\
\text { Garment industry } \\
\mathrm{F}=10.7 \% ; \text { Shoe } \\
\text { and leather } \\
\text { industry } \\
\mathrm{F}=50.0 \% \text {; } \\
\text { Manufacture of } \\
\text { wood and wood } \\
\text { products } \\
\mathrm{F}=33.3 \% ; \text { Pulp, } \\
\text { paper and paper } \\
\text { products } \\
\text { manufacturing } \\
\mathrm{F}=11.1 \% ; \\
\text { Publishing, } \\
\text { printing and } \\
\text { reproduction of } \\
\text { recorded media } \\
\mathrm{F}=50.0 \% ; \\
\text { Chemical industry } \\
\mathrm{F}=33.3 \% ; \\
\text { Manufacture of } \\
\text { rubber and plastic } \\
\text { products } \\
\mathrm{F}=0.0 \% ; \\
\text { Manufacture of } \\
\text { non-metallic min- } \\
\text { eral products } \\
\mathrm{F}=50.0 \% ; \\
\text { Manufacture of } \\
\text { basic metals } \\
\mathrm{F}=18.2 \% ; \\
\text { Manufacture of } \\
\text { fabricated metal } \\
\text { products } \\
\mathrm{F}=23.1 \% ;\end{array}$ \\
\hline
\end{tabular}


Table 1 Qualitative overview of prevalence of WMSDs in secondary industries of Europe (Continued)

\begin{tabular}{|c|c|c|c|c|}
\hline $\begin{array}{l}\text { Author, year, } \\
\text { study design, } \\
\text { industry \& country }\end{array}$ & $\begin{array}{l}\text { Period of } \\
\text { measurement }\end{array}$ & $\begin{array}{l}\text { Demographic } \\
\text { characteristics }\end{array}$ & $\begin{array}{l}\text { Examination } \quad \text { Tools for examination } \\
\text { method \& } \\
\text { response rate }\end{array}$ & $\begin{array}{l}\text { WMSD \& } \\
\text { Prevalence }\end{array}$ \\
\hline & & $\begin{array}{l}\text { metal products } \\
\mathrm{F}=26 ; \\
\text { Manufacture of } \\
\text { electrical and } \\
\text { optical } \\
\text { equipment } \\
\mathrm{NEC} \\
\mathrm{F}=69 ; \\
\text { Manufacture of } \\
\text { electrical and } \\
\text { optical } \\
\text { equipment } \\
\mathrm{F}=2 ; \\
\text { Manufacture of } \\
\text { motor vehicles } \\
\mathrm{F}=2 ; \\
\text { Manufacture of } \\
\text { other transport } \\
\text { equipment } \\
\mathrm{F}=45 ; \\
\text { Manufacture of } \\
\text { furniture and } \\
\text { wood } \\
\text { industries } \\
\mathrm{F}=0 ; \\
\text { Recycling } \\
\mathrm{M}=182 ; \text { Food } \\
\text { and drink } \\
\text { industry } \\
\mathrm{M}=1 ; \\
\text { Garment } \\
\text { industry } \\
\mathrm{M}=8 \text {; Shoe } \\
\text { and leather } \\
\text { industry } \\
\mathrm{M}=24 ; \\
\text { Manufacture of } \\
\text { wood and } \\
\text { wood } \\
\text { products } \\
\mathrm{M}=52 ; \text { Pulp, } \\
\text { paper and } \\
\text { paper products } \\
\text { manufacture } \\
\mathrm{M}=17 ; \\
\text { Publishing, } \\
\text { printing, } \\
\text { reproduction } \\
\text { of recorded } \\
\text { media } \\
\mathrm{M}=8 ; \\
\text { Chemical } \\
\text { industry } \\
\mathrm{M}=84 ; \\
\text { Manufacture of } \\
\text { rubber and } \\
\text { plastic } \\
\text { products } \\
\mathrm{M}=22 ; \text { Non- } \\
\text { metallic min- } \\
\text { eral products } \\
\text { manufacturing } \\
\mathrm{M}=23 ; \\
\text { Manufacture of } \\
\text { basic metals } \\
\mathrm{M}=91 ;\end{array}$ & & $\begin{array}{l}\text { Manufacture of } \\
\text { electrical and } \\
\text { optical } \\
\text { equipment NEC } \\
\mathrm{F}=14.5 \% ; \\
\text { Manufacture of } \\
\text { electrical and } \\
\text { optical } \\
\text { equipment } \\
\mathrm{F}=0.0 \% \text {; } \\
\text { Manufacture of } \\
\text { motor vehicles } \\
\mathrm{F}=50.0 \% ; \\
\text { Manufacture of } \\
\text { other transport } \\
\text { equipment } \\
\mathrm{F}=17.8 \% ; \\
\text { Manufacture of } \\
\text { furniture and } \\
\text { wood industries } \\
\mathrm{F}=0 \% ; \text { Recycling } \\
\mathrm{M}=12.1 \% ; \text { Food } \\
\text { and drink industry } \\
\mathrm{M}=0 \% ; \text { Garment } \\
\text { industry } \\
\mathrm{M}=0 \% \text {; Shoe and } \\
\text { leather industry } \\
\mathrm{M}=12.5 \% ; \\
\text { Manufacture of } \\
\text { wood and wood } \\
\text { products } \\
\mathrm{M}=11.5 \% ; \\
\text { Manufacture of } \\
\text { pulp, paper and } \\
\text { paper products } \\
\mathrm{M}=5.9 \% ; \\
\text { Publishing, } \\
\text { printing and } \\
\text { reproduction of } \\
\text { recorded media } \\
\mathrm{M}=0.0 \% ; \\
\text { Chemical industry } \\
\mathrm{M}=14.3 \% ; \\
\text { Manufacture of } \\
\text { rubber and plastic } \\
\text { products } \\
\mathrm{M}=4.6 \% ; \text { Non- } \\
\text { metallic mineral } \\
\text { products } \\
\text { manufacturing } \\
\mathrm{M}=8.7 \% ; \\
\text { Manufacture of } \\
\text { basic metals } \\
\mathrm{M}=18.7 \% ; \\
\text { Manufacture of } \\
\text { fabricated metal } \\
\text { products } \\
\mathrm{M}=14.6 \% ; \\
\text { Manufacture of } \\
\text { electrical and } \\
\text { optical } \\
\text { equipment NEC } \\
\mathrm{M}=15.7 \% ; \\
\text { Manufacture of } \\
\text { electrical and } \\
\text { optical }\end{array}$ \\
\hline
\end{tabular}


Table 1 Qualitative overview of prevalence of WMSDs in secondary industries of Europe (Continued)

\begin{tabular}{|c|c|c|c|c|c|}
\hline $\begin{array}{l}\text { Author, year, } \\
\text { study design, } \\
\text { industry \& country }\end{array}$ & $\begin{array}{l}\text { Period of } \\
\text { measurement }\end{array}$ & $\begin{array}{l}\text { Demographic } \\
\text { characteristics }\end{array}$ & $\begin{array}{l}\text { Examination } \\
\text { method \& } \\
\text { response rate }\end{array}$ & Tools for examination & $\begin{array}{l}\text { WMSD \& } \\
\text { Prevalence }\end{array}$ \\
\hline & & $\begin{array}{l}\text { Manufacture of } \\
\text { fabricated } \\
\text { metal products } \\
M=89 ; \\
\text { Manufacture of } \\
\text { electrical and } \\
\text { optical } \\
\text { equipment } \\
N E C \\
M=89 ; \\
\text { Manufacture of } \\
\text { electrical and } \\
\text { optical } \\
\text { equipment } \\
M=63 ; \\
\text { Manufacture of } \\
\text { motor vehicles } \\
M=91 ; \\
\text { Manufacture of } \\
\text { other transport } \\
\text { equipment } \\
M=57 ; \\
\text { Manufacture of } \\
\text { furniture and } \\
\text { wood } \\
\text { industries } \\
M=7 ; \\
\text { Recycling }\end{array}$ & & & $\begin{array}{l}\text { equipment } \\
M=25.4 \% ; \\
\text { Manufacture of } \\
\text { motor vehicles } \\
M=11.1 \% ; \\
\text { Manufacture of } \\
\text { other transport } \\
\text { equipment } \\
M=10.5 \% ; \\
\text { Manufacture of } \\
\text { furniture and } \\
\text { wood industries } \\
M=0 \% \text {; Recycling }\end{array}$ \\
\hline $\begin{array}{l}\text { Harkness et al. } 2003 \text { [30] } \\
\text { Cohort } \\
\text { Shipyard industry - UK }\end{array}$ & $\begin{array}{l}\text { Not } \\
\text { mentioned } \\
\text { Prevalence } \\
\text { Period: } \\
1 \text { month }\end{array}$ & $\begin{array}{l}T=82 ; 12- \\
\text { month follow- } \\
\text { up } \\
T=67 ; 24- \\
\text { month follow- } \\
\text { up }\end{array}$ & $\begin{array}{l}\text { Self-report } \\
86 \% ; 12 \text {-month } \\
\text { follow-up } \\
\text { 92\%; 24-month } \\
\text { follow-up }\end{array}$ & $\begin{array}{l}\text { Questionnaire for pain involving one } \\
\text { question: "Thinking back over the } \\
\text { past month, have you had any ache } \\
\text { or pain which lasted for one day or } \\
\text { longer?' If so, indicate the site of this } \\
\text { pain on a line drawing of the body." }\end{array}$ & $\begin{array}{l}\text { Shoulder } T=11 \% \\
(n=9) ; 12 \text {-month } \\
\text { follow-up } \\
\text { Shoulder } T=19 \% \\
(n=13) ; 24- \\
\text { month follow-up }\end{array}$ \\
\hline $\begin{array}{l}\text { Hussain } 2004[31] \\
\text { Cross-sectiona } \\
\text { Transport industry - UK }\end{array}$ & $\begin{array}{l}\text { Not } \\
\text { mentioned } \\
\text { Prevalence } \\
\text { Period: } \\
12 \text { months }\end{array}$ & $\begin{array}{l}T=323 ; \bar{y}= \\
36.5 \text { years }\end{array}$ & $\begin{array}{l}\text { Self-report } \\
70 \%\end{array}$ & $\begin{array}{l}\text { Modified version of the Nordic } \\
\text { Musculoskeletal Questionnaire }\end{array}$ & $\begin{array}{l}\text { Neck T=60\% } \\
(n=194) \\
\text { Shoulder T }=57 \% \\
(n=184) \\
\text { Upper Back T = } \\
17 \%(n=55) \\
\text { Elbow T }=20 \% \\
(n=65) \\
\text { Lower Back T = } \\
65 \%(n=211) \\
\text { Wrist/Hand T= } \\
46 \%(n=149) \\
\text { Hip T = 8\% ( } n= \\
26) \\
\text { Knee T }=39 \% \\
(n=126) \\
\text { Ankle/Foot T }= \\
13 \%(n=42)\end{array}$ \\
\hline $\begin{array}{l}\text { Isolani et al. } 2002 \text { [32] } \\
\text { Cross-sectional } \\
\text { Food industry - Italy }\end{array}$ & $\begin{array}{l}\text { March-May } \\
1998 \\
\text { Prevalence } \\
\text { Period: } \\
\text { Point } \\
\text { prevalence }\end{array}$ & $\begin{array}{l}T=114 ; \bar{y}= \\
38.0 \text { years } \\
F=21(18 \%) ; \\
22-61 \text { years } \\
M=93(82 \%) ; \\
22-61 \text { years }\end{array}$ & $\begin{array}{l}\text { Trained physicians } \\
71 \%\end{array}$ & $\begin{array}{l}\text { Interview } \\
\text { Physical examination } \\
\text { Median NCSs }\end{array}$ & $\begin{array}{l}\text { Carpal tunnel } \\
\text { syndrome T = } \\
53 \%(n=60)\end{array}$ \\
\hline $\begin{array}{l}\text { Kaergaard and Andersen } 2000 \\
\text { [33] } \\
\text { Cohort } \\
\text { Textile industry - Denmark }\end{array}$ & $\begin{array}{l}\text { 1994-1997 } \\
\text { Prevalence } \\
\text { Period: } \\
\text { Point } \\
\text { prevalence }\end{array}$ & $\begin{array}{l}F=243 ; \bar{y}= \\
38.3 \text { years }\end{array}$ & $\begin{array}{l}\text { Self-report } \\
\text { Trained physicians } \\
\text { who were blinded } \\
\text { to the answers } \\
\text { from the } \\
\text { questionnaire }\end{array}$ & $\begin{array}{l}\text { Questionnaire about current } \\
\text { musculoskeletal complaints } \\
\text { Physical examination (neck and } \\
\text { arms) }\end{array}$ & $\begin{array}{l}\text { Neck/Shoulder } \\
T=77.6 \% ; \\
\text { baseline } \\
\text { Myofascial pain } \\
\text { syndrome T= } \\
15.2 \% ; \text { baseline }\end{array}$ \\
\hline
\end{tabular}


Table 1 Qualitative overview of prevalence of WMSDs in secondary industries of Europe (Continued)

\begin{tabular}{|c|c|c|c|c|}
\hline $\begin{array}{l}\text { Author, year, } \\
\text { study design, } \\
\text { industry \& country }\end{array}$ & $\begin{array}{l}\text { Period of } \\
\text { measurement }\end{array}$ & $\begin{array}{l}\text { Demographic } \\
\text { characteristics }\end{array}$ & $\begin{array}{l}\text { Examination } \\
\text { method \& } \\
\text { response rate }\end{array}$ & Tools for examination \\
\hline & & & $\begin{array}{l}\text { 94\%; baseline } \\
\text { 55\%; follow-up }\end{array}$ & \\
\hline $\begin{array}{l}\text { Le Manac'h et al. } 2012 \text { [34] } \\
\text { Cross-sectional } \\
\text { Food, metal, electronic industry - } \\
\text { France }\end{array}$ & $\begin{array}{l}\text { April 2002- } \\
\text { April } 2005 \\
\text { Prevalence } \\
\text { Period: } \\
12 \text { months }\end{array}$ & $\begin{array}{l}T=295 ; \text { Food } \\
\text { industry } \\
T=102 ; \text { Metal } \\
\text { industry } \\
T=115 ; \\
\text { Electronic } \\
\text { industry } \\
\bar{y}=38.7 \text { years }\end{array}$ & $\begin{array}{l}\text { Self-report } \\
\text { Occupational } \\
\text { physicians } \\
93 \% \text { of all workers }\end{array}$ & $\begin{array}{l}\text { Nordic Auto-Questionnaire } \\
\text { Physical examination, if indicated }\end{array}$ \\
\hline $\begin{array}{l}\text { Leclerc et al. } 2001 \text { [52] } \\
\text { Cohort } \\
\text { Electrical, textile, food, and } \\
\text { packaging industry - France }\end{array}$ & $\begin{array}{l}\text { 1993-1994 } \\
\text { 1996-1997 } \\
\text { Prevalence } \\
\text { Period: } \\
\text { Point } \\
\text { prevalence }\end{array}$ & $\begin{array}{l}\mathrm{T}=247 \\
\text { Electrical } \\
\text { industry } \\
\mathrm{T}=63 ; \text { Textile } \\
\text { industry } \\
\mathrm{T}=143 ; \text { Food } \\
\text { industry } \\
\mathrm{T}=103 ; \\
\text { Packaging }\end{array}$ & $\begin{array}{l}\text { Self-report } \\
\text { Occupational } \\
\text { physicians } \\
42.11 \%\end{array}$ & $\begin{array}{l}\text { Self-Administered Questionnaire } \\
\text { Physical examination }\end{array}$ \\
\hline
\end{tabular}

WMSD \&

Prevalence

Rotator cuff

tendinitis $\mathrm{T}=$

5.8\%; baseline

Knee bursitis $\mathrm{T}=$

$1.4 \%(n=4)$; Food industry

Knee bursitis $T=$

$2.0 \%(n=2)$; Metal industry

Knee bursitis $\mathrm{T}=$

$0.9 \%(n=1)$;

Electronic

industry

Carpal tunnel syndrome $T=$ $21.9 \%(n=54)$.

Electronic

industry

Carpal tunnel syndrome $\mathrm{T}=$

$27.0 \%(n=17)$;

Textile industry

Carpal tunnel

syndrome $\mathrm{T}=$

13.3\% ( $n=19)$;

Food industry

Carpal tunnel

syndrome $\mathrm{T}=$

$31.1 \%(n=32)$;

Packaging

Lateral

epicondylitis $\mathrm{T}=$

$20.2 \%(n=50)$

Electronical

industry

Lateral

epicondylitis $\mathrm{T}=$ $4.8 \%(n=3)$;

Textile industry

Lateral

epicondylitis $\mathrm{T}=$

$7.0 \%(n=10)$;

Food industry

Lateral

epicondylitis $\mathrm{T}=$

7.8\% $(n=8)$;

Packaging

Wrist tendinitis

$T=8.5(n=21)$;

Electronical

industry

Wrist tendinitis

$\mathrm{T}=3.2 \%(n=2)$

Textile industry

Wrist tendinitis

$\mathrm{T}=21.0 \%(n=30)$;

Food industry

Wrist tendinitis

$\mathrm{T}=10.7 \%(n=11)$;

Packaging

Lima et al. 2019 [35]

Cross-sectional

Food industry - Portugal
May 2016

Prevalence

Period:

12 months
$T=20$ (27\%).

24-33 years

$\mathrm{T}=21$ (28\%);

34-44 years

$\mathrm{T}=17$ (23\%);
Self-report

$35.23 \%$
Nordic Musculoskeletal

Questionnaire
Neck $T=15 \%$

$(n=3) ; 24-33$

years

Neck $T=29 \%$

$(n=6) ; 34-44$ 
Table 1 Qualitative overview of prevalence of WMSDs in secondary industries of Europe (Continued)

\begin{tabular}{lll}
\hline $\begin{array}{l}\text { Author, year, } \\
\text { study design, } \\
\text { industry \& country }\end{array}$ & $\begin{array}{l}\text { Period of } \\
\text { measurement }\end{array}$ & $\begin{array}{l}\text { Demographic } \\
\text { characteristics }\end{array}$ \\
\hline & $44-52$ years \\
& $T=16(22 \%) ;$ \\
$54-63$ years \\
\\
$F=53(71.6 \%)$ \\
$M=21(28.4 \%)$ \\
$y=41.9$ years
\end{tabular}

Nordander et al. 2008 [36]

Cross-sectional

Manufacturing industry and mechanical assembly industry -

Sweden
Not

mentioned

Prevalence

Period:

7 days
$\mathrm{F}=172 ; \bar{y}=$ 42.0 years $\mathrm{M}=105 ; \mathrm{y}=$ 36.0 years
Examiners not specified

Not mentioned
Questionnaire-based interview Physical examination (modified scheme by Ohlsson et al.)
WMSD \&

Prevalence

years

Neck $\mathrm{T}=65 \%$

$(\mathrm{n}=11)$; 44-53

years

Neck $\mathrm{T}=63 \%$

$(n=10) ; 54-63$

years

Lower Back $\mathrm{T}=$

$35 \%(n=7) ; 24$

33 years

Lower Back $\mathrm{T}=$

$19 \%(n=4) ; 34$

44 years

Lower Back $\mathrm{T}=$

$35 \%(n=6) ; 44$

53 years

Lower Back $\mathrm{T}=$

$69 \%(n=11) ; 54-$

63 years

Right Shoulder

$T=30 \%(n=6)$;

24-33 years

Right Shoulder

$\mathrm{T}=24 \%(\mathrm{n}=5)$;

34-44 years

Right Shoulder

$T=53 \%(n=9)$;

44-53 years

Right Shoulder

$T=38 \%(n=6)$;

54-63 years

Neck/Shoulder

$\mathrm{F}=61 \%(n=104)$

Elbow/Hand $\mathrm{F}=$

$55 \%(n=95)$

Lower Back F =

$30 \%(n=52)$

Knee/Foot $\mathrm{F}=$

$41 \%(n=70)$

Tension neck

syndrome $\mathrm{F}=$

$20 \%(n=34)$

Cervicalgia $\mathrm{F}=6 \%$

$(n=11)$

Shoulder

tendinitis $F=16 \%$

$(n=27)$

Acromioclavicular

syndrome $\mathrm{F}=$

$12 \%(n=20)$

Epicondylitis $F=$ $6 \%(n=11)$

Pronator teres

syndrome $\mathrm{F}=2 \%$

$(n=3)$

Radial tunnel

syndrome $\mathrm{F}=1 \%$

$(n=1)$

Carpal tunnel

syndrome $\mathrm{F}=8 \%$

$(n=13)$

Overused hand

syndrome $\mathrm{F}=2 \%$

$(n=3)$

Peritendinitis/

Tenosynovitis $\mathrm{F}=$ 
Table 1 Qualitative overview of prevalence of WMSDs in secondary industries of Europe (Continued)

Author, year, study design, industry \& country
Period of Demographic Examination

measurement characteristics method \& response rate

WMSD \&

Prevalence

$7 \%(n=12)$

De Quervain's

disease $\mathrm{F}=1 \%$

$(\mathrm{n}=2)$

Neck/Shoulder

$M=36 \%(n=38)$

Elbow/Hand $\mathrm{M}=$

$34 \%(n=36)$

Lower Back $\mathrm{M}=$

$27 \%(n=28)$

Knee/Foot $M=$

$32 \%(n=34)$

Tension neck

syndrome $\mathrm{M}=$

$12 \%(n=13)$

Cervicalgia $M=$

$3 \%(n=3)$

Shoulder

tendinitis $M=6 \%$

$(n=6)$

Acromioclavicular syndrome $M=7 \%$

$(n=7)$

Epicondylitis $\mathrm{M}=$

$3 \%(n=3)$

Pronator teres

syndrome $\mathrm{M}=0 \%$

$(n=0)$

Radial tunnel

syndrome $\mathrm{M}=0 \%$

$(n=0)$

Carpal tunnel

syndrome $M=2 \%$

$(\mathrm{n}=2)$

Overused hand

syndrome $M=0 \%$

$(n=0)$

Peritendinitis/

Tenosynovitis

$M=3 \%(n=3)$

De Quervain's

disease $M=0 \%$

$(\mathrm{n}=0)$

Ólafsdóttir and Rafnsson 2000

[37]

Cross-sectional

Fish industry - Iceland

\begin{tabular}{|c|c|}
\hline $\begin{array}{l}\text { Not } \\
\text { mentioned } \\
\text { Prevalence } \\
\text { Period: } \\
\text { 1) } 12 \text { months } \\
\text { 2) } 7 \text { days }\end{array}$ & $\begin{array}{l}T=254 \\
F=49(19 \%) ; \\
16-19 \text { years } \\
F=80(32 \%) ; \\
20-29 \text { years } \\
F=125(49 \%) ; \\
30-54 \text { years }\end{array}$ \\
\hline
\end{tabular}

Neck $=69 \% ; 12-$

month

prevalence

Shoulder $=78 \%$;

12-month

prevalence

Elbow =17\%; 12 -

month

prevalence

Wrist $=47 \%$; 12 -

month

prevalence

Upper Back =

37\%; 12-month

prevalence

Lower Back =

68\%; 12-month

prevalence

Hip $=22 \%$; 12 -

month

prevalence

Knee $=28 \%$; 12 -

month 
Table 1 Qualitative overview of prevalence of WMSDs in secondary industries of Europe (Continued)

Author, year, Period of Demographic Examination Tools for examination

WMSD \&

Prevalence

Pope et al. 2001 [38]

Cross-sectional

Packaging industry - UK

Ricco and Signorelli 2017 [53]

Cross-sectional

Meat industry - Italy

Roquelaure et al. 2002 [54]

Cohort

Shoe industry - France

\begin{tabular}{|c|c|c|c|}
\hline $\begin{array}{l}\text { Not } \\
\text { mentioned } \\
\text { Prevalence } \\
\text { Period: } \\
1 \text { month }\end{array}$ & $T=203$ & $\begin{array}{l}\text { Self-report } \\
83 \% \text { of all workers }\end{array}$ & $\begin{array}{l}\text { Manikin and Shoulder Disability } \\
\text { Questionnaire }\end{array}$ \\
\hline $\begin{array}{l}\text { January } 2012 \\
\text { December } \\
2013 \\
\text { Prevalence } \\
\text { Period: } \\
\text { Point } \\
\text { prevalence }\end{array}$ & $\begin{array}{l}T=434 ; \bar{y}= \\
37.0 \text { years } \\
F=198(45.6 \%) \\
M=236 \\
(54.4 \%)\end{array}$ & $\begin{array}{l}\text { Trained clinician } \\
91.8 \%\end{array}$ & $\begin{array}{l}\text { Full medical assessment } \\
\text { Ultrasonography and/or NCS in } \\
\text { clinically possible cases }\end{array}$ \\
\hline $\begin{array}{l}\text { 1996-1997 } \\
\text { Prevalence } \\
\text { Period: } \\
12 \text { months }\end{array}$ & $\begin{array}{l}\text { Year 1996: } \\
T=253 ; \bar{y}= \\
40.2 \text { years } \\
F=158(62 \%) \\
M=95(38 \%) \\
\text { Year 1997: } \\
T=191 ; \bar{y}= \\
41.1 \text { years } \\
F=117(61 \%) \\
M=74(39 \%)\end{array}$ & $\begin{array}{l}\text { Occupational } \\
\text { physicians } \\
90 \%\end{array}$ & $\begin{array}{l}\text { Interview } \\
\text { Physical examination }\end{array}$ \\
\hline
\end{tabular}

prevalence

Ankle $=24 \%$; $12-$

month

prevalence

Head $=56 \%$; $12-$

month

prevalence

Fingers $=31 \%$; 12

month

prevalence

Neck $=44 \%$; 7-day

prevalence

Shoulder $=47 \%$;

7-day prevalence

Elbow $=9 \%$; 7-day

prevalence

Wrist $=26 \%$; 7-day

prevalence

Upper Back =

18\%; 7-day

prevalence

Lower Back =

39\%; 7-day

prevalence

Hip $=16 \%$; 7-day

prevalence

Knee $=15 \%$; 7-day prevalence

Ankle $=15 \%$; 7 -

day prevalence

Head = 39\%; 7-

day prevalence

Fingers $=19 \%$; 7 -

day prevalence

Shoulder $T=27 \%$ $(n=55)$

Carpal tunnel syndrome $\mathrm{F}=$ $17.2 \%(n=34)$

Carpal tunnel syndrome $M=$

$11.4 \%(n=27)$

Tension neck syndrome $\mathrm{T}=$ $7.5 \%(n=19)$;

1996

Rotator cuff

syndrome $=7.9 \%$

$(\mathrm{n}=20) ; 1996$

Medial

epicondylitis $\mathrm{T}=$

0\% $(n=0) ; 1996$

Lateral

epicondylitis $T=$

2.0\% ( $n=5) ; 1996$

Cubital tunnel

syndrome $\mathrm{T}=$

$5.2 \%(n=10)$;

1996

Radial tunnel

syndrome $T=$ 
Table 1 Qualitative overview of prevalence of WMSDs in secondary industries of Europe (Continued)

Author, year, Period of Demographic Examination Tools for examination

WMSD \&

Prevalence

$\begin{array}{ll}\text { April to } & \mathrm{F}=69 ; \text { Food } \\ \text { September } & \text { industry } \\ 2002 & \mathrm{~F}=31 ; \text { Textile } \\ \text { May to } & \text { industry } \\ \text { October } 2003 & \mathrm{~F}=5 ; \text { Wood } \\ \text { Prevalence } & \text { industry } \\ \text { Period: } & \mathrm{F}=20 ; \text { Paper } \\ \text { Not specified } & \text { industry } \\ & \mathrm{F}=39 ; \\ & \text { Chemical } \\ & \text { industry } \\ & \mathrm{F}=9 ; \text { Steel } \\ & \text { industry } \\ & \mathrm{F}=22 ; \\ & \text { Machine and } \\ & \text { equipment } \\ & \text { industry } \\ & \mathrm{F}=32 ; \\ & \text { Computer } \\ & \text { industry } \\ & \mathrm{F}=3 ; \\ & \text { Automotive } \\ & \text { industry } \\ \mathrm{F}=43 ; \\ \mathrm{Furniture} \\ \text { industry } \\ \end{array}$

$0.4 \%(n=1) ; 1996$

Carpal tunnel syndrome $\mathrm{T}=$

$18.2 \%(n=46)$

1996

Guyon's canal syndrome $T=$ $0.4 \%(n=1) ; 1996$ Hand/Wrist tendinitis $T=2.4 \%$ $(\mathrm{n}=6) ; 1996$ Tension neck syndrome $\mathrm{T}=$ 4.2\% ( $(n=8) ; 1997$ Rotator cuff syndrome $\mathrm{T}=$ 9.5\% $(n=18)$; 1997

Medial epicondylitis $\mathrm{T}=$ $0.5 \%(n=1) ; 1997$ Lateral epicondylitis $\mathrm{T}=$ $3.1 \%(n=6) ; 1997$

Cubital tunnel syndrome $\mathrm{T}=$ 4.2\% ( $n=8) ; 1997$ Radial tunnel syndrome $\mathrm{T}=$ $1.0 \%(n=2) ; 1997$

Carpal tunnel syndrome $\mathrm{T}=$ $22.0 \%(n=42)$ 1997

Guyon's canal syndrome $\mathrm{T}=$ $0.5 \%(n=1) ; 1997$ Hand/Wrist tendinitis $\mathrm{T}=3.1 \%$ $(n=6) ; 1997$

Roquelaure et al. 2006 [39]
Cross-sectional
Food, textile, wood, paper,
chemical, steel, machine and
equipment, computer,
automotive, furniture and wood
industry - France

Roquelaure et al. 2006 [39]

Food, textile, wood, paper automotive, furniture and wood industry - France

Occupational
physicians
$17.4 \%$ of the
Sentinal network
occupational
physicians

Nordic Musculoskeletal

Questionnaire

VAS

If symptoms occurred during the past 12 months: physical examination based on the criteria document for the evaluation of work-related upper-limb MSDs
Upper extremity disorders

$F=15.9 \%(n=11)$; Food industry $F=19.4 \%(n=6) ;$ Textile industry

$\mathrm{F}=/(\mathrm{n}=2)$; Wood industry $F=25.0 \%(n=5)$; Paper industry $\mathrm{F}=35.9 \%(n=14) ;$ Chemical industry $F=33.3 \%(n=3)$; Steel industry $F=27.3 \%(n=6)$; Machine and equipment industry $F=12.5 \%(n=4)$;

Computer industry $\mathrm{F}=/(\mathrm{n}=1)$; Automotive industry $\mathrm{F}=20.9 \%(n=9)$; Furniture industry 
Table 1 Qualitative overview of prevalence of WMSDs in secondary industries of Europe (Continued)

\begin{tabular}{|c|c|c|c|c|c|}
\hline $\begin{array}{l}\text { Author, year, } \\
\text { study design, } \\
\text { industry \& country }\end{array}$ & $\begin{array}{l}\text { Period of } \\
\text { measurement }\end{array}$ & $\begin{array}{l}\text { Demographic } \\
\text { characteristics }\end{array}$ & $\begin{array}{l}\text { Examination } \\
\text { method \& } \\
\text { response rate }\end{array}$ & Tools for examination & $\begin{array}{l}\text { WMSD \& } \\
\text { Prevalence }\end{array}$ \\
\hline & & $\begin{array}{l}F=112 ; \text { Food } \\
\text { industry } \\
F=112 ; \text { Textile } \\
\text { industry } \\
F=23 \text {; Wood } \\
\text { industry } \\
F=55 ; \text { Paper } \\
\text { industry } \\
F=74 \text {, } \\
\text { Chemical } \\
\text { industry } \\
F=81 ; \text { Steel } \\
\text { industry } \\
F=72 ; \\
\text { Machine and } \\
\text { equipment } \\
\text { industry } \\
F=51 ; \\
\text { Computer } \\
\text { industry } \\
F=40 ; \\
\text { Automotive } \\
\text { industry } \\
F=50 ; \\
\text { Furniture } \\
\text { industry }\end{array}$ & & & $\begin{array}{l}M=12.5 \%(n= \\
14) ; \text { Food industry } \\
M=0 \%(n=0) ; \\
\text { Textile industry } \\
M=13 \%(n=3) ; \\
\text { Wood industry } \\
M=10.9 \%(n=6) ; \\
\text { Paper industry } \\
M=12.2 \%(n=9) ; \\
C h e m i c a l \text { industry } \\
M=14.8 \%(n= \\
12) ; \text { Steel industry } \\
M=12.5 \%(n=9) ; \\
\text { Machine and } \\
\text { equipment } \\
\text { industry } \\
M=7.8 \%(n=4) ; \\
\text { Computer } \\
\text { industry } \\
M=20 \%(n=8) ; \\
\text { Automotive } \\
\text { industry } \\
M=20.0 \%(n=5) ; \\
\text { Furniture industry }\end{array}$ \\
\hline $\begin{array}{l}\text { Sormunen et al. } 2009 \text { [40] } \\
\text { Cross-sectional } \\
\text { Meat and dairy industry - Finland }\end{array}$ & $\begin{array}{l}1997 \\
\text { Prevalence } \\
\text { Period: } \\
12 \text { months }\end{array}$ & $\begin{array}{l}T=117 \\
F=46 \% ; \bar{y}= \\
35.0 \text { years } \\
M=54 \% ; \bar{y}= \\
33.0 \text { years }\end{array}$ & $\begin{array}{l}\text { Self-report } \\
85 \%\end{array}$ & Questionnaire & $\begin{array}{l}\text { Neck/Shoulder } \\
\mathrm{F}=89 \%(n=424) ; \\
18-64 \text { years } \\
\text { Neck/Shoulder } \\
\mathrm{F}=90 \%(n=275) ; \\
18-39 \text { years } \\
\text { Neck/Shoulder } \\
\mathrm{F}=86 \%(n=149) ; \\
49-64 \text { years } \\
\text { Shoulder } \mathrm{F}=65 \% \\
(n=291) ; 18-64 \\
\text { years } \\
\text { Shoulder } \mathrm{F}=60 \% \\
(n=178) ; 18-39 \\
\text { years } \\
\text { Shoulder } \mathrm{F}=75 \% \\
(n=113) ; 49-64 \\
\text { years } \\
\text { Lower Back F= } \\
68 \%(n=303) ; \\
18-64 \text { years } \\
\text { Lower Back F= } \\
65 \%(n=193) ; \\
18-39 \text { years } \\
\text { Lower Back F= } \\
73 \%(n=109) ; \\
49-64 \text { years } \\
\text { Wrist F = 71\% } \\
(n=318) ; 18-64 \\
\text { years } \\
\text { Wrist F }=70 \% \\
(n=213) ; 18-39 \\
\text { years } \\
\text { Wrist F }=72 \% \\
(n=105) ; 49-64 \\
\text { years } \\
\text { Neck/Shoulder } \\
\text { M=78\% }(n= \\
432) ; 18-64 \text { years } \\
\text { Neck/Shoulder }\end{array}$ \\
\hline
\end{tabular}


Table 1 Qualitative overview of prevalence of WMSDs in secondary industries of Europe (Continued)

\begin{tabular}{|c|c|c|c|c|c|}
\hline $\begin{array}{l}\text { Author, year, } \\
\text { study design, } \\
\text { industry \& country }\end{array}$ & $\begin{array}{l}\text { Period of } \\
\text { measurement }\end{array}$ & $\begin{array}{l}\text { Demographic } \\
\text { characteristics }\end{array}$ & $\begin{array}{l}\text { Examination } \\
\text { method \& } \\
\text { response rate }\end{array}$ & Tools for examination & $\begin{array}{l}\text { WMSD \& } \\
\text { Prevalence }\end{array}$ \\
\hline & & & & & $\begin{array}{l}\mathrm{M}=75 \%(\mathrm{n}= \\
318) ; 18-39 \text { years } \\
\text { Neck/Shoulder } \\
\mathrm{M}=87 \%(\mathrm{n}= \\
113) ; 49-64 \text { years } \\
\text { Shoulder M=57\% } \\
(n=305) ; 18-64 \\
\text { years } \\
\text { Shoulder M=50\% } \\
(n=204) ; 18-39 \\
\text { years } \\
\text { Shoulder M = 82\% } \\
(n=99) ; 49-64 \\
\text { years } \\
\text { Lower Back M= } \\
64 \%(n=347) ; \\
18-64 \text { years } \\
\text { Lower Back M = } \\
60 \%(n=253) ; \\
18-39 \text { years } \\
\text { Lower Back M= } \\
75 \%(n=92) ; 49- \\
64 \text { years } \\
\text { Wrist M = 58\% } \\
(n=311) ; 18-64 \\
\text { years } \\
\text { Wrist M=57\% } \\
(n=237) ; 18-39 \\
\text { years } \\
\text { Wrist M =63\% } \\
(n=74) ; 49-64 \\
\text { years }\end{array}$ \\
\hline $\begin{array}{l}\text { Sundstrup et al. } 2014 \text { [41] } \\
\text { Cross-sectional } \\
\text { Meat industry - Denmark }\end{array}$ & $\begin{array}{l}\text { Not } \\
\text { mentioned } \\
\text { Prevalence } \\
\text { Period: } \\
3 \text { months }\end{array}$ & $\begin{array}{l}T=595 ; \bar{y}= \\
44.0 \text { years } \\
F=11 \% \\
M=89 \%\end{array}$ & $\begin{array}{l}\text { Self-report } \\
92 \%\end{array}$ & $\begin{array}{l}\text { Questionnaire: 0-10 modified VAS } \\
\text { scale for neck, shoulder, elbow, and } \\
\text { hand/wrist regions defined by } \\
\text { drawings from the Nordic } \\
\text { Musculoskeletal Questionnaire. }\end{array}$ & $\begin{array}{l}\text { Neck } T=48 \% \\
\text { Shoulder } T=60 \% \\
\text { Elbow } T=40 \% \\
\text { Hand/Wrist T= } \\
52 \%\end{array}$ \\
\hline $\begin{array}{l}\text { Wixted et al. } 2018[42] \\
\text { Cross-sectiona } \\
\text { Manufacturing industry - Ireland }\end{array}$ & $\begin{array}{l}\text { Not } \\
\text { mentioned } \\
\text { Prevalence } \\
\text { Period: } \\
12 \text { months }\end{array}$ & $\begin{array}{l}T=47(20 \%) ; \\
21-30 \text { years } \\
T=97(41.3 \%) ; \\
31-40 \text { years } \\
T=(27.7 \%) ; \\
41-50 \text { years } \\
T=(11.1 \%) ; \\
51-60 \text { years } \\
F=47(20 \%) \\
M=188(80 \%)\end{array}$ & $\begin{array}{l}\text { Self-report } \\
80 \%\end{array}$ & $\begin{array}{l}\text { Nordic Musculoskeletal } \\
\text { Questionnaire (Amended) }\end{array}$ & $\begin{array}{l}\text { Neck } \mathrm{T}=41 \% \\
(n=96) \\
\text { Shoulder T=46\% } \\
(n=108) \\
\text { Upper back T }= \\
28 \%(n=66) \\
\text { Lower back T }= \\
57 \%(n=134)\end{array}$ \\
\hline $\begin{array}{l}\text { Weyh et al. } 2020 \text { [43] } \\
\text { Cross-sectional } \\
\text { Steel industry - Germany }\end{array}$ & $\begin{array}{l}\text { July } 2016 \\
\text { to May } 2017 \\
\text { Prevalence } \\
\text { Period: } \\
12 \text { months }\end{array}$ & $\begin{array}{l}T=145 ; \bar{y}= \\
35.8 \text { years } \\
F=2(1 \%) \\
M=143(99 \%)\end{array}$ & $\begin{array}{l}\text { Self-report } \\
\text { Not mentioned }\end{array}$ & $\begin{array}{l}\text { Modified version of the Nordic } \\
\text { Musculoskeletal Questionnaire }\end{array}$ & $\begin{array}{l}\text { Neck } \mathrm{T}=61 \% \\
\text { Shoulder } \mathrm{T}=55 \% \\
\text { Elbow } \mathrm{T}=32 \% \\
\text { Forearm } \mathrm{T}=32 \% \\
\text { Upper back T = } \\
36 \% \\
\text { Lower back T }= \\
71 \% \\
\text { Hip }=9 \% \\
\text { Knee }=44 \% \\
\text { Lower leg }=16 \%\end{array}$ \\
\hline
\end{tabular}


analysis) and will be discussed in more detail with their corresponding prevalence data (Table 1).

\section{Quantitative analysis}

Seventeen of the 28 studies were selected based on aforementioned eligibility criteria [20-24, 26, 27, 30, 31, $33,35-38,40,42,43]$.

12-month prevalence Twelve studies [20-24, 26, 31, $35,37,40,42,43$ ] were divided in 12 subgroups of WMSDs: neck, shoulder, shoulder/neck, elbow, wrist/ hand, upper back, lower back, back (for studies that did not make a distinction between upper or lower back), hip, knee, ankle/feet, leg (for studies that did not make a distinction between hip, knee or ankle/feet). The heterogeneity analysis illustrated a considerable heterogeneity for the majority of subgroups (neck, shoulder, shoulder/ neck, wrist/hand, back, upper back, lower back, leg and knee) with ranges between 76 and 96\%. A substantial heterogeneity was obtained for the hip subgroup $\left(\mathrm{I}^{2}=\right.$ $63 \%)$ and both the elbow and ankle/feet subgroups displayed a heterogeneity of non-importance $\left(\mathrm{I}^{2}=5 \%\right.$ and $\mathrm{I}^{2}=36 \%$, respectively) [55]. Due to the lack of sufficient articles, additional subgroup analyses could not be performed to further investigate these high heterogeneity values. Pooled sample sizes of 9540, 7464, 1097, 2984, 4507, 5405, 788, 4922, 1023, 5710, 643 and 788 bluecollar secondary industry workers were obtained for back, shoulder/neck, neck, shoulder, lower back, wrist/ hand, knee, leg, upper back, elbow, ankle/feet and hip subgroups, respectively. A distinct distribution of WMSDs over time could not be identified, however the food industry was the industry generally most represented in all subgroups. On average, industrial workers had the most WMSDS in the back (back: mean 60\%; SD 13\%, lower back: mean 47\%; SD 20\%), shoulder (shoulder: mean 50\%; SD 18\%, shoulder/neck: mean 54\%; SD
27\%) and neck regions (mean 51\%; SD 15\%). Hip complaints were the least prevalent (mean 11\%; SD 7\%) (Table 2).

A mean of $60 \%$ (range 38-72\%; SD 13\%) back WMSDs was reported in secondary industries (Fig. 2). The food industry was the industry most represented in this analysis [20, 24, 35, 40]. Lower back WMSDs obtained a mean prevalence of $47 \%$ (range $24-71 \%$; SD 20\%) and were researched in the food, transport, shipyard, production, textile, manufacturing and steel industry (Figure B supplementary material). With the exception of the production industry, where Andersen et al. [23] reported both baseline and follow-up prevalence data, all industries were represented equally [21-23, 31, 37, 42, 43]. Upper back WMSDs were prevalent in $22 \%$ (range 6$37 \%$; SD 12) of the investigated pooled sample size with food [37], transport [31], textile [21], manufacturing [42] and steel [43] industries included in the subgroup analysis (Figure $\mathrm{C}$ supplementary material).

Shoulder/neck WMSDs obtained a mean prevalence of 54\% (range 19-83\%; SD 27\%) and were reported in the food [20, 24, 40], shipyard [22] and production [23] industry (Fig. 3). Isolated neck WMSDs were prevalent in $51 \%$ (range $32-69 \%$, SD 15\%) of the secondary industrial workers (Figure D supplementary material) and isolated shoulder WMSDs in 50\% (range 23-78\%; SD 18\%) of the workers researched (Figure E supplementary material). In both subgroups, the food industry was the industry sector most represented $[35,37,40]$ and also the transport [31], textile [21], manufacturing [42] and steel [43] industry were included in the analysis.

Wrist/hand WMSDs, with a mean prevalence of $42 \%$ (range 14-64\%; SD 16\%) were investigated in the food [20, 24, 37, 40], transport [31], shipyard [22] and textile [21] industry (Fig. 4). Further, a prevalence of $29 \%$ (range 16-56\%; SD 16\%) was obtained for overall leg WMSDs in food [20, 24], production [23] and steel

Table 2 Overall prevalence of WMSDs per anatomical region

\begin{tabular}{|c|c|c|c|c|c|}
\hline Anatomical location & Mean (\%) with $95 \% \mathrm{Cl}$ & Median (\%) & Range (\%) & $S D(\%)$ & $I^{2}(\%)$ \\
\hline Back & $60[49 ; 70]$ & 66 & $38-72$ & 13 & 83 \\
\hline Shoulder/Neck & $54[31 ; 74]$ & 51 & $19-83$ & 27 & 96 \\
\hline Neck & $51[40 ; 62]$ & 51 & $32-69$ & 15 & 86 \\
\hline Shoulder & $50[37 ; 63]$ & 51 & $23-78$ & 18 & 91 \\
\hline Lower back & $47[33 ; 61]$ & 47 & $24-71$ & 20 & 93 \\
\hline Wrist/Hand & $42[31 ; 54]$ & 44 & $14-64$ & 16 & 90 \\
\hline Knee & $33[24 ; 43]$ & 34 & $21-46$ & 11 & 76 \\
\hline Leg & $29[18 ; 43]$ & 23 & $16-56$ & 16 & 89 \\
\hline Upper back & $22[13 ; 37]$ & 28 & $6-37$ & 12 & 90 \\
\hline Elbow & $21[18 ; 24]$ & 21 & $15-30$ & 4 & 5 \\
\hline Ankle/Feet & $17[12 ; 23]$ & 15 & $13-24$ & 6 & 36 \\
\hline Hip & $11[7 ; 18]$ & 9 & $8-22$ & 7 & 63 \\
\hline
\end{tabular}




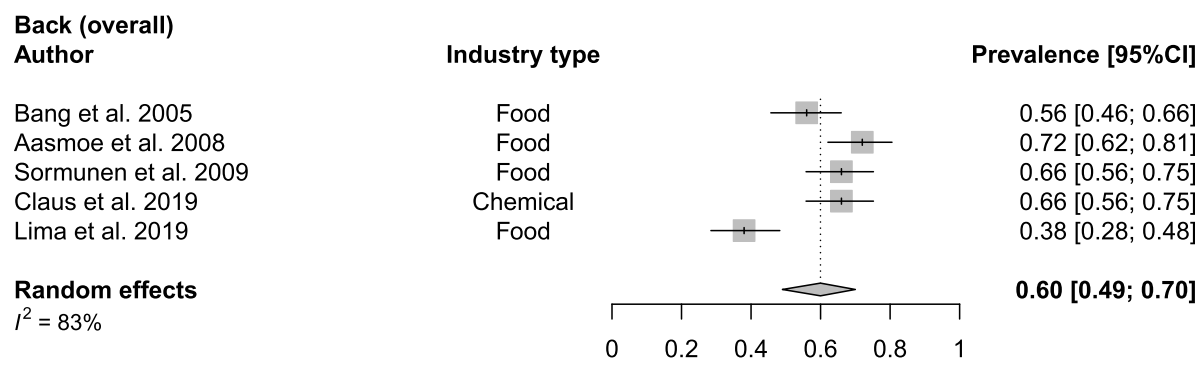

Fig. 2 Meta-analytic overview of prevalence of back WMSDs

industries [43] (Fig. 5). Considering studies that differentiate between hip, knee and ankle/feet WMSDs, mean prevalence values were obtained of $11 \%$ (range 8-22; SD 7\%), 33\% (range 21-46\%; SD 11\%) and 17\% (range 1324\%; SD 6\%), respectively (Figure F, G, H supplementary material).

Food [20, 24, 37], transport [31], production [23], textile [21] and steel [43] industries were all affected by elbow WMSDs with a prevalence mean of $21 \%$ (range 15-30\%; SD 4\%) (Fig. 6).

3-month prevalence Sundstrup et al. [41] reported 3month prevalence values of $60,52,48,40 \%$ for the shoulder, wrist/hand, neck and elbow, respectively. No further analysis was performed due to the lack of sufficient articles.

1-month prevalence Two studies reported a 1-month prevalence value for shoulder WMSDs with a calculated mean prevalence of $22 \%$ (range 11-27\%; SD 8\%) $[30,38]$.

7-day prevalence WMSDs during a 7-day period were researched in three studies [26, 36, 37]. Prevalence values of $47,44,26,18,16$ and $15 \%$ for shoulder, neck, wrist/hand, upper back, hip and ankle feet WMSDs, respectively, were solely researched in one study [37]. Overall back WMSDs with a prevalence of $26 \%$ were reported by [26] and neck/shoulder WMSDs with a prevalence of $52 \%$ were discussed by Nordander et al. [36].
Lower back, elbow and knee WMSDs were both researched in two studies and mean prevalence values of 34\% (range 29-39\%; SD 7\%), 29\% (range 9-47\%; SD $27 \%$ ) and 27\% (range 15-38\%; SD 16\%), respectively, were obtained [36, 37].

Point-prevalence De Zwart et al. [27] investigated different secondary industry sectors of which mean pointprevalence values of $16 \%$ (range 11-20\%; SD 3\%), 11\% (range 7-15\%; SD 3\%), 5\% (range 4-6\%; SD 0\%) and 5\% (range 3-6\%; SD 1\%) were obtained for shoulder, neck, wrist/hand and elbow WMSDs, respectively. Kaergaard and Andersen [33] also investigated point-prevalence, however they focused on the textile industry and shoulder/neck WMSDs where a prevalence of $78 \%$ was reported.

\section{Incidence}

\section{Qualitative analysis}

The majority of studies were published between 2000 and 2010 [44-49, 51, 52, 54]. Four countries and ten different industries were obtained. Similar to prevalence data, cohort and cross-sectional study designs were preferred in incidence research with nine [44-50, 52, 54] and two studies [51, 53], respectively. Further, the majority of studies reported a response rate greater than $50 \%[44,46-48,53,54]$ and included sample sizes between 100 and 500 participants which is in line with included prevalence studies [48-50, 52-54]. Further, evaluation of WMSDs was primarily performed through
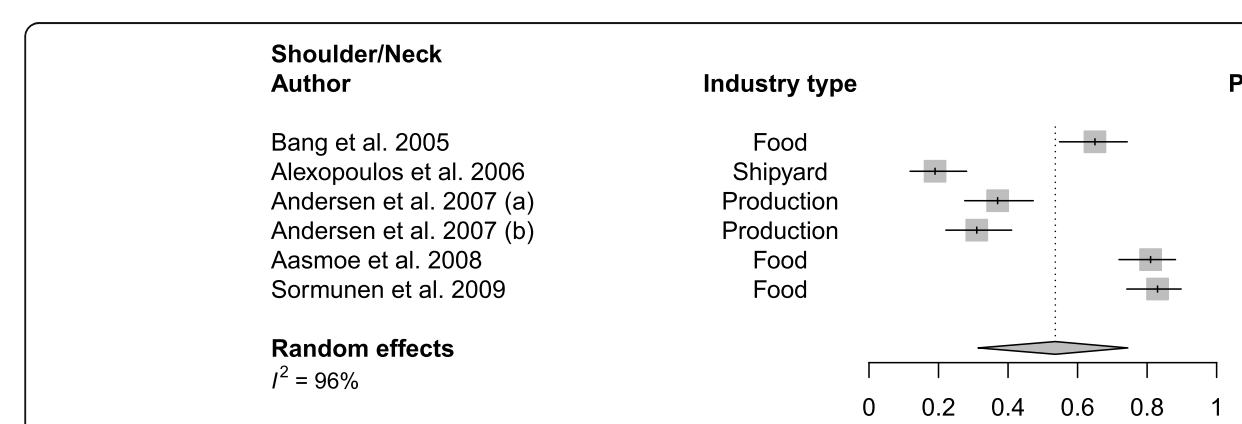

Prevalence $[95 \% \mathrm{Cl}]$

$0.65[0.55 ; 0.74]$

$0.19[0.12 ; 0.28]$

$0.37[0.28 ; 0.47]$

$0.31[0.22 ; 0.41]$

$0.81[0.72 ; 0.88]$

$0.83[0.74 ; 0.90]$

$0.54[0.31 ; 0.74]$

Fig. 3 Meta-analytic overview of prevalence of shoulder/neck WMSDs. (a) = baseline; (b) = 24-month follow-up 


Wrist/Hand
Author
Ólafsdóttir and Rafnsson 2000 (a)
Ólafsdóttir and Rafnsson 2000 (b)
Hussain 2004
Bang et al. 2005
Alexopoulos et al. 2006
Aasmoe et al. 2008
Sormunen et al. 2009
Afonso et al. 2014
Random effects
$I^{2}=90 \%$

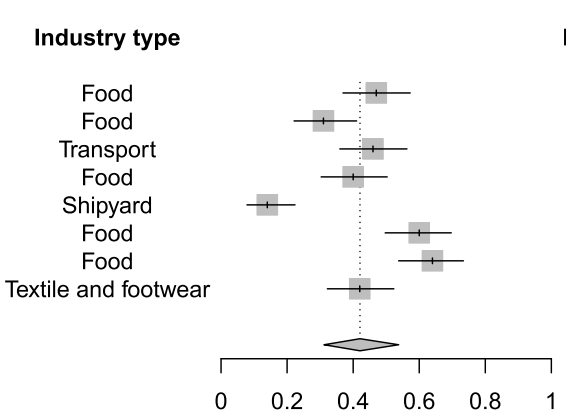

Prevalence $[95 \% \mathrm{Cl}]$

$0.47[0.37 ; 0.57]$

$0.31[0.22 ; 0.41]$

$0.46[0.36 ; 0.56]$

$0.40[0.30 ; 0.50]$

$0.14[0.08 ; 0.22]$

$0.60[0.50 ; 0.70]$

$0.64[0.54 ; 0.73]$

$0.42[0.32 ; 0.52]$

$0.42[0.31 ; 0.54]$

Fig. 4 Meta-analytic overview of prevalence of wrist/hand WMSDs. $(a)=$ wrist; $(b)=$ fingers

a combination of reviewing obtained questionnaire data and conducting physical examinations [48, 51, 52, 54]. All studies reported the employment of a health professional to identify WMSDs [44-49, 51, 52, 54] (Table 3).

Upper limb and neck/back disorders were the most commonly studied WMSDs with 11 [44-54] and 6 [44$47,49,54]$ studies, respectively, reporting incidence data. A further discrimination in upper limb WMSDs was made by five studies [47, 51-54] with carpal tunnel syndrome being the most frequently reported upper limb disorder [51-54]. Leclerc et al. [52] and Roquelaure et al. [54] both documented epicondylitis lateralis and wrist tendinitis as upper limb WMSDs. In addition, three studies reported incidence data regarding lower limb disorders without further anatomical details [4446]. Overall, incidence ratios were reported or calculated for six of the eleven studies [48-50, 52-54]. Calculations could not be performed for other studies due to the lack of sample size descriptions. Overall, incidence ratios of upper limb WMSDs ranged from 0.04 to 0.26 [48-50]. A maximum ratio of 0.14 was reported for carpal tunnel syndrome [53], minimum ratios of 0.12 were stated by Leclerc et al. [52] and Roquelaure et al. [54]. Two authors documented incidence ratios regarding lateral epicondylitis and wrist tendinitis of 0.13 and 0.06 , respectively, and 0.02 and 0.03 , respectively [52, 54]. Häkkänen et al. [49] stated an overall incidence ratio of 0.19 for back WMSDs and 0.14 for neck/shoulder disorders (Table 3).

\section{Discussion}

The goal of this meta-analysis was to provide insight on the prevalence and incidence of WMSDs in Europe's secondary industries. Results showed that back (overall), shoulder/neck, neck, shoulder, lower back and wrist WMSDs were the most prevalent with mean values of 60\% (range 38-72\%), 54\% (range 18-83\%), 51\% (range 32-69\%), 50\% (range 23-78\%), 47\% (range 24-71\%) and $42 \%$ (range 14-64\%), respectively. Incidence ratios of upper limb disorders were the most common reported and ranged from 0.04 to 0.26 [48-50]. The food industry was the most prominent researched sector for the prevalence of back (overall), elbow, leg (overall), shoulder, neck, shoulder/neck, and wrist/hand WMSDs subgroups. This specific industry type was frequently associated with high prevalence values of WMSDs [20, 24, 35, $37,40]$. The food sector is often characterised by working in a cold environment, which could form an additional risk factor for developing WMSDs [20, 24, 40].

\section{Back WMSDs}

Overall back WMSDs obtained the highest prevalence values of all subgroups (60\%). This finding is corroborated by the European Agency for Safety and Health at Work (EU-OSHA) with a 12-month prevalence value of $55 \%$ reported in industrial workers (i.e. plant and machine operators, assemblers), indicating the significant susceptibility of this population for developing back WMSDs [1]. Further, lower back WMSDs were more

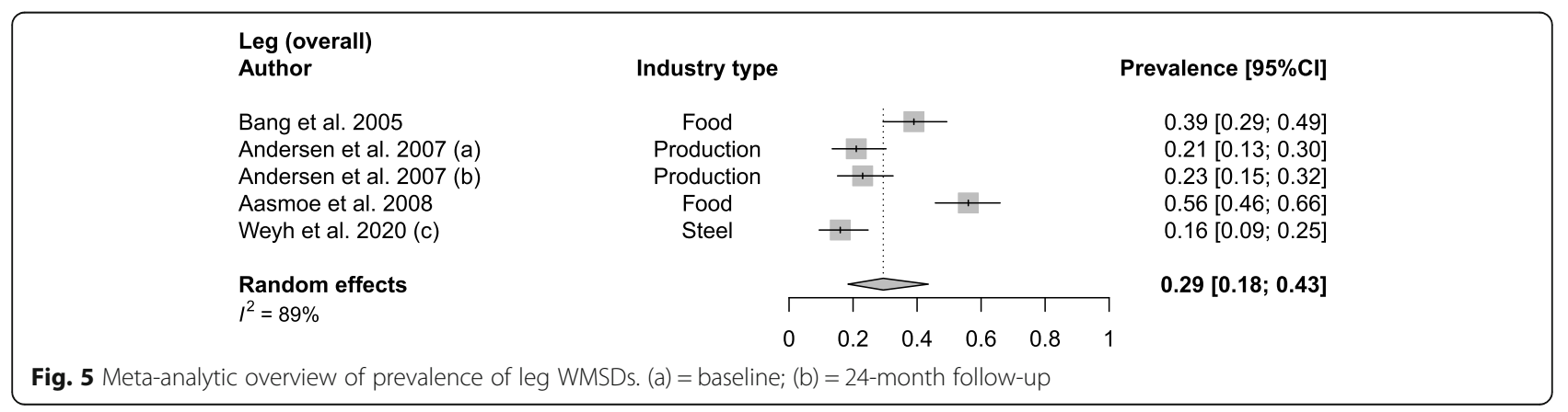




$\begin{aligned} & \text { Elbow } \\ & \text { Author }\end{aligned}$
Ólafsdóttir and Rafnsson 2000
Hussain 2004
Bang et al. 2005
Andersen et al. 2007 (a)
Andersen et al. 2007 (b)
Aasmoe et al. 2008
Afonso et al. 2014
Weyh et al. 2020
Random effects
$I^{2}=5 \%$

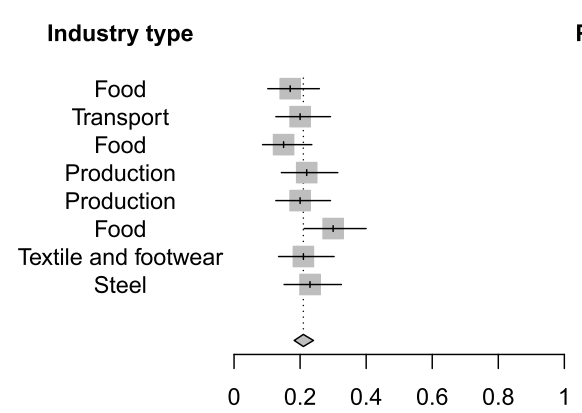

Prevalence $[95 \% \mathrm{Cl}]$

$0.17[0.10 ; 0.26]$

$0.20[0.13 ; 0.29]$

$0.15[0.09 ; 0.24]$

$0.22[0.14 ; 0.31]$

$0.20[0.13 ; 0.29]$

$0.30[0.21 ; 0.40]$

$0.21[0.13 ; 0.30]$

$0.23[0.15 ; 0.32]$

$0.21[0.18 ; 0.24]$

Fig. 6 Meta-analytic overview of prevalence of elbow WMSDs. (a) = baseline; $(b)=24$-month follow-up

frequently reported (47\%) compared to upper back WMSDs (22\%). This corresponds to values reported in the systematic review of Briggs et al. [56] where a 12month prevalence range between 3 to $55 \%$ and medians around 30\% were obtained for upper back WMSDs in most occupational groups. This difference between prevalence of lower and upper back WMSDs may explain the growing interest in lower back preventive measures since this anatomical location is clearly more prone to develop WMSDs.

It is assumed that the presence of biomechanical risk factors (e.g. lifting heavy loads or performing repetitive task) and psychosocial risk factors (e.g. low job control or level of support from colleagues) increases the development of WMSDs $[2,57]$. This correlation was found in the majority of studies $[20,35,37,42]$. Therefore, to effectively prevent this type of WMSDs, implementation of robotic devices e.g. exoskeletons or collaborative robots could offer great potential by reducing biomechanical loads. Further, studies implementing the Nordic musculoskeletal questionnaire (NMQ) reported higher prevalence values in the lower and upper back subgroup $[22,26,31,35,37,42,43]$. It is possible that this examining method provided a more thorough self-evaluation than non-validated questionnaires. However, this was not the case for the overall back WMSDs subgroup. Since other factors could have influenced these results (e.g. characteristics of sample size, presence of risk factors, etc.) and high heterogeneity was obtained for all back WMSDs subgroups, it is recommended to interpret these observations with caution.

Regarding incidence of back WMSDs, Häkkänen et al. [49] reported incidence ratios of 0.19 for males (indicating that over the course of 1 year 19 out of 100 persons reported new back WMSDs) and 0.24 for females working in the transport industry. This indicates a relative limited development of new back WMSDs between 1987 and 1990. A European report of 2005 described relatively similar 1-year incidence ratios of low back WMSDs that ranged between 0.12 and 0.29 for Austria and 0.28 for the Czech Republic [58]. These relatively low incidence values and high prevalence values indicate a limited onset of back WMSDs with long-term complaints indicating the need for effective prevention strategies. Nevertheless, studies researching incidence of (lower) back WMSDs in secondary industries of twenty-first century Europe are scarce and results should be interpreted with caution.

\section{Upper limb and neck WMSDs}

The neck, shoulder, and neck/shoulder subgroup obtained the highest prevalence values of upper limb WMSDs with 51, 50 and 54\%, respectively. These findings are in line with the work of Buckle and Devereux [59]. No clear trends in prevalence data could be observed in studies reporting high psychosocial stress or the presence of biomechanical risk factors. Although the exact reason for this observation remains speculative, it would not be surprising to find the cause in the known high heterogeneity. Also no discrepancies were found between studies that obtained prevalence data through validated questionnaires, non-validated questionnaires or expert assessment. An exception was the neck subgroup where the NMQ tends to result in higher prevalence values. It is possible that the thoroughness of this questionnaire led to this observation. However, since only one study in this subgroup refrained from using the NMQ [21], under-representation of other questionnaires could also have attributed to finding this trend. The need for effective prevention strategies is again highlighted in these subgroups. Implementation of new robotic devices to optimise employee's ergonomics and therefore reduce risks for developing WMSDs form an extremely promising strategy to combat WMSDs in the future $[12,13]$.

Incidence of upper limb WMSDs was researched the most. Between 1987 and 1990, incidence ratios of 0.13 were reported (indicating that over the course of 1 year 13 out of 100 persons reported new upper limb WMSDs) [49]. Later from 1996 to 1997 and from 1997 to 2000, incidence ratios of 0.26 and 0.8 were obtained, respectively [48]. These results 
Table 3 Qualitative overview of incidence of WMSDs in secondary industries of Europe

Author, year, study design, period of measurement, industry \& country

Chen et al. 2006 [44]

Cohort: 1996-2001

Food and organic products, petrochemical, metals, and automotive industry - UK
Cherry et al. 2000 [47]

\section{Cohort: 1996-2001}

Food and organic products, petrochemical, metals, and automotive industry - UK
Occupational physicians

Not further specifie

$75 \%$ of occupational physicians of OPRA

\section{Cherry et al. 2001 [46]}

Cohort: October 1997-September 2000

Food and organic products, petrochemical, metals, and automotive industry - UK and Scotland
Examination method, response rate

Occupational physicians Not further specified $75 \%$ of occupational physicians of OPRA
Not

mentioned

(n)

Demographic
characteristics

WSMD \&

Incidence cases/ Incidence ratio

Upper limb = 1827/

FOM

Neck/Back = 792/

million persons-year;

FOM

Lower $\operatorname{limb}=121 /$

million persons-year;

FOM

Upper limb = 1945/

million persons-year; PRP

Neck/Back = 517/

million persons-year; PRP

Lower limb = 49/

million persons-year;

PRP

Upper limb = 5618/

million persons-year; MAP

Neck/Back = 1886/

million persons-year; MAP

Lower limb = 317/

million persons-year; MAP

Hand/Wrist/Arm =

708; FOM

Hand/Wrist/Arm =

960; PRP

Hand/Wrist/Arm =

1092; MM

Hand/Wrist/Arm =

1164; AM

Lumbar Spine/

Trunk = 384; FOM

Lumbar Spine/

Trunk = 276; PRP

Lumbar Spine/

Trunk = 300; MM

Lumbar Spine/

Trunk =684; AM

Elbow $=264 ; \mathrm{FOM}$

Elbow $=348 ;$ PRP

Elbow $=348 ; \mathrm{MM}$

Elbow = 516; AM

Shoulder = 300; AM

Rheumatologists

Not further specified

$80 \%$ of the society's

membership of

rheumatologists.
Not

mentioned

\section{Overall}

incidence ratio

Could not be

calculated

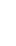


Table 3 Qualitative overview of incidence of WMSDs in secondary industries of Europe (Continued)

\section{Author, year, study design, period of measurement,} industry \& country

$\begin{array}{ll}\text { Examination method, } & \begin{array}{l}\text { Demographic } \\ \text { examination tools \& } \\ \text { response rate }\end{array}\end{array}$

response rate

Occupational physicians Not further specified

Cohort: 1996-2001

Food and organic material manufacturing, petrochemical, rubber Not mentioned and plastics manufacturing, metallic and automotive products manufacturing - UK and Scotland
Not mentioned
Descatha et al. 2007 [48]

Cohort: 1996-2000

Shoe industry - France
Occupational physician Interview

Physical examination $66 \%$

In
In

Neck/Back $=51 ;$ PRP
Neck/Back $=30 ;$ MM
Neck/Back $=12 ;$ AM

Upper limb $F=217 / \quad$ Could not be million persons-year; calculated FOM

Upper limb F = 342/

million persons-year; PRP

Upper limb $F=598 /$

million persons-year; MAP

Back $F=48 /$ million

persons-year; FOM

Back $F=172 /$ million

persons-year; MAP

Upper limb $M=$

106/million persons-

year; FOM

Upper limb $M=$

104/million personsyear; PRP

Upper limb $M=$

443/million persons-

year; MAP

Back $M=79 /$ million

persons-year; FOM

Back $M=42 /$ million

persons-year; PRP

Back $M=153$ /

million persons-year;

MAP

Lower Limb $M=28$ /

million persons-year; MAP

$T=166$
$T=66.3 \% ;<$
45 years
$T=33.7 \% ; \geq$
45 years
$F=60.8 \%$
$M=39.2 \%$

UWMSD

$\mathrm{F}=0.238$; 1996

1997

$M=0.296$; $1996-$

1997

$\mathrm{T}<45 \mathrm{y}=0.23$;

1996-1997

$\mathrm{T} \geq 45$ years $=0.33$;

1996-1997

$F=0,08 ; 1997-2000$

$M=0.08 ; 1997-2000$

$\mathrm{T}<45 \mathrm{y}=0.08$;

1997-2000

$T \geq 45$ years $=0.09$;

1997-2000

0.26 ; 1996-

1997

0.08 ; $1997-$

2000

$$
\begin{aligned}
& \text { Carpal tunnel } \\
& \text { syndrome } \\
& F=38 ; F D I \\
& F=13 ; G I \\
& F=30 ; S L I \\
& F=1 ; \mathrm{WW} \\
& F=1 ; P P P \\
& F=5 ; P P R \\
& F=12 ; C l \\
& F=6 ; P R P \\
& F=0 ; \mathrm{NMM} \\
& F=3 ; \mathrm{BM} \\
& F=7 ; \mathrm{FMP} \\
& F=0 ; E O N \\
& F=23 ; E O
\end{aligned}
$$

Overall

incidence ratio

\footnotetext{
Food and drink, garment, shoe and leather, manufacture of ment, manufacture of motor vehicles, manufacture of other transport equipment, manufacture of furniture and wood, recycling industry - France
}

$\begin{array}{ll}\text { Neurologists } & F=113 ; \mathrm{FDI} \\ \text { Occupational physicians } & \mathrm{F}=12 ; \mathrm{Gl} \\ \text { Physical examinatio } & \mathrm{F}=28 ; \mathrm{SLI} \\ \text { 17.4\% of the occupational } & \mathrm{F}=6 ; \mathrm{WW} \\ \text { physicians } & \mathrm{F}=12 ; \mathrm{PPP} \\ \mathrm{F} & =9 ; \mathrm{PPR} \\ \mathrm{F} & =2 ; \mathrm{Cl} \\ \mathrm{F} & =45 ; \mathrm{PRP} \\ \mathrm{F} & =2 ; \mathrm{NMM} \\ \mathrm{F} & =6 ; \mathrm{BM} \\ \mathrm{F} & =11 ; \mathrm{FMP} \\ \mathrm{F} & =26 ; \mathrm{EON} \\ \mathrm{F} & =69 ; \mathrm{EO} \\ \mathrm{F} & =2 ; \mathrm{MM} \\ \mathrm{F} & =2 ; \mathrm{MOT}\end{array}$

Could not be calculated 
Table 3 Qualitative overview of incidence of WMSDs in secondary industries of Europe (Continued)

Author, year, study design, period of measurement, industry \& country
Häkkänen et al. 2001 [49]

Cohort: January 1987-September 199

Transport industry - Finland
Leclerc et al. 2001 [52]

Cohort: 1993-1994

1996-1997

Electrical, textile, food, and packaging industry - France
Monaco et al. 2019 [50]

Retrospective cohort: January 2012-December 2015

Transport industry - Italy
Occupational physicians Physical examination Not mentioned examination tools \& response rate

$\begin{aligned} & \text { Demographic } \\ & \text { characteristics }\end{aligned}$
$F=45 ; \mathrm{FWI}$
$F=0 ; \mathrm{R}$
$M=182 ; \mathrm{FDI}$
$M=1 ; \mathrm{Gl}$
$M=8 ; \mathrm{SLI}$
$M=24 ; \mathrm{WW}$
$M=52 ; \mathrm{PPP}$
$M=17 ; \mathrm{PPR}$
$M=8 ; \mathrm{Cl}$
$M=84 ; \mathrm{PRP}$
$M=22 ; \mathrm{NMM}$
$M=23 ; \mathrm{BM}$
$M=91 ; \mathrm{FMP}$
$M=89 ; \mathrm{EON}$
$M=89 ; \mathrm{EO}$
$M=63 ; \mathrm{MM}$
$M=91 ; \mathrm{MOT}$
$M=57 ; \mathrm{FWI}$
$M=7 ; \mathrm{R}$

$M=7 ; R$

$\mathrm{T}=364$

$F=55$

$M=309$
Self-report

Occupational health physician

Self-Administered

Questionnaire

Physical examination $42.11 \%$

$T=247 ; E O$
$T=63 ; T I$
$T=143 ; F I$
$T=103 ; P$

\section{WSMD \& \\ Overall \\ Incidence cases/ \\ incidence} Incidence ratio

$\mathrm{F}=12 ; \mathrm{MM}$

$\mathrm{F}=0 ; \mathrm{MOT}$

$F=9 ; F W I$

$\mathrm{F}=1 ; \mathrm{R}$

$M=22 ; F D I$

$M=1 ; G \mid$

$M=11 ; S L I$

$M=6 ; W W$

$\mathrm{M}=1 ; \mathrm{PPP}$

$\mathrm{M}=2 ; \mathrm{PPR}$

$\mathrm{M}=2 ; \mathrm{Cl}$

$M=5 ; P R P$

$\mathrm{M}=0 ; \mathrm{NMM}$

$M=6 ; B M$

$M=15 ; F M P$

$\mathrm{M}=7$; EON

$\mathrm{M}=8$; $\mathrm{EO}$

$M=10 ; M M$

$\mathrm{M}=0$; MOT

$M=9 ; \mathrm{FWI}$

$M=0 ; R$

Upper Limb $F=0.2$

Not mentioned

Reviewing medical

records of health

surveillance visits

Not mentioned Neck/Shoulder $\mathrm{F}=$

0.35

Lower Back F $=0.24$ Upper Limb M =

0.11

Necks

0.10

Lower Back M = 0.19

Carpal Tunnel Could not be Syndrome $=0.04 ; \mathrm{EO}$ calculated Carpal Tunnel

Syndrome $=0.06 ; \mathrm{Tl}$

Carpal Tunnel

Syndrome $=0.05 ; \mathrm{FI}$

Carpal Tunnel

Syndrome $=0.03 ; \mathrm{P}$

Lateral

Epicondylitis $=0.06$

EO

Lateral

Epicondylitis $=0.03$;

$\mathrm{TI}$

Lateral

Epicondylitis $=0.04$

Lateral

Epicondylitis $=0.03$; P

Wrist Tendinitis $=$

0.01; EO

Wrist Tendinitis $=$

0.02; $\mathrm{TI}$

Wrist Tendinitis $=$

0.03; FI

Wrist Tendinitis $=$

0.03; P

Upper $\operatorname{limb}=$

Shoulder =

0.14

Lower Back = 0.19 (n)

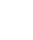

Upper Limb T $=0.04 \quad 0.04$
$\mathrm{T}=171$
$T=78$ (45.6\%);
25-34 years
$\mathrm{T}=60$ (35.1\%);
35-44 years
$\mathrm{T}=33$ (19.3\%):
$\geq 45$ years


Table 3 Qualitative overview of incidence of WMSDs in secondary industries of Europe (Continued)

\begin{tabular}{|c|c|c|c|c|}
\hline $\begin{array}{l}\text { Author, year, study design, period of measurement, } \\
\text { industry \& country }\end{array}$ & $\begin{array}{l}\text { Examination method, } \\
\text { examination tools \& } \\
\text { response rate }\end{array}$ & $\begin{array}{l}\text { Demographic } \\
\text { characteristics }\end{array}$ & $\begin{array}{l}\text { WSMD \& } \\
\text { Incidence cases/ } \\
\text { Incidence ratio }\end{array}$ & $\begin{array}{l}\text { Overall } \\
\text { incidence } \\
\text { ratio }\end{array}$ \\
\hline & & $\begin{array}{l}F=14(8.2 \%) \\
M=157 \\
(91.8 \%)\end{array}$ & & \\
\hline $\begin{array}{l}\text { Ricco and Signorelli } 2017 \text { [53] } \\
\text { Cross-sectional: } \\
\text { January } 2012 \\
\text { December } 2013 \\
\text { Meat industry - Italy }\end{array}$ & $\begin{array}{l}\text { Trained clinician } \\
\text { Full medical assessment } \\
\text { Ultrasonography and/or } \\
\text { NCS in clinically possible } \\
\text { cases } \\
91.8 \%\end{array}$ & $\begin{array}{l}T=434 ; \bar{y}= \\
37.0 \text { years } \\
F=198(45.6 \%) \\
M=236 \\
(54.4 \%)\end{array}$ & $\begin{array}{l}\text { Carpal tunnel } \\
\text { syndrome } \\
\mathrm{F}=0.21 \\
\mathrm{M}=0.11\end{array}$ & 0.14 \\
\hline $\begin{array}{l}\text { Roquelaure et al. } 2002 \text { [54] } \\
\text { Cohort: 1996-1997 } \\
\text { Shoe industry - France }\end{array}$ & $\begin{array}{l}\text { Occupational physicians } \\
\text { Interview } \\
\text { Physical examination } \\
90 \%\end{array}$ & $\begin{array}{l}\text { Year 1996: } \\
T=253 ; \bar{y}= \\
40.2 \text { years } \\
F=158(62 \%) \\
M=95(38 \%) \\
Y \text { Year } 1997: \\
T=191 ; \bar{y}= \\
41.1 \text { years } \\
F=117(61 \%) \\
M=74(39 \%)\end{array}$ & $\begin{array}{l}\text { Tension Neck } \\
\text { Syndrome T=0.04 } \\
(n=7) ; 1997 \\
\text { Rotator Cuff } \\
\text { Syndrome T=0.06 } \\
(n=12) ; 1997 \\
\text { Medial Epicondylitis } \\
T=0 ; 1997 \\
\text { Lateral Epicondylitis } \\
\mathrm{T}=0.02(n=4) ; 1997 \\
\text { Cubital Tunnel } \\
\text { Syndrome T=0.03 } \\
(n=5) ; 1997 \\
\text { Radial Tunnel } \\
\text { Syndrome T }=0.01 \\
(n=2) ; 1997 \\
\text { Carpal Tunnel } \\
\text { Syndrome T }=0.12 \\
(n=23) ; 1997 \\
\text { Guyon's Canal } \\
\text { Syndrome T }=0.005 \\
(n=1) ; 1997 \\
\text { Hand-Wrist } \\
\text { Tendinitis T }=0.03 \\
(n=6) ; 1997\end{array}$ & $\begin{array}{l}\text { Lateral } \\
\text { epicondylitis = } \\
0.02 \\
\text { Carpal Tunnel } \\
\text { Syndrome = } \\
0.12 \\
\text { Wrist } \\
\text { Tendinitis = } \\
0.03\end{array}$ \\
\hline
\end{tabular}

FOM Food and organic material manufacture, $P R P$ Petrochemical, rubber and plastics manufacture, MAP Metallic and automotive products manufacture, MM Metals manufacture, AM Automotive manufacture, FDI Food and drink industry, GI Garment industry, SLI Shoe and leather industry, WW Manufacture of wood and wood products, PPP Manufacture of pulp, paper and paper products, PPR Publishing, printing and reproduction of recorded media, CI Chemical industry, NMM Manufacture of other non-metallic mineral products, BM Manufacture of basic metals, FMP Manufacture of fabricated metal products, EON Manufacture of electrical and optical equipment not elsewhere classified, EO Manufacture of electrical and optical equipment, MM Manufacture of motor vehicles, MOT Manufacture of other transport equipment, FWI Manufacture of furniture and wood industries, R Recycling, $T I$ Textile industry; FI Food industry, $P$ Packaging, $T$ Total, $F$ Females, $M$ Males, $\bar{y}$ Mean age, / = Not reported

carefully suggest that the occurrence of new upper limb WMSDs decreases over time in secondary industries. However, some caution when interpreting these results is advised since no other studies were included to support these statements. Monaco et al. [50] reported an incidence ratio of 0.04 between 2012 and 2015, implying a further decline of upper limb WMSDs. This is contradicted by data provided by EU-OSHA stating that a significant reduction in upper limb WMSDs incidence data is still absent over the beginning course of the twenty-first century [58]. Lower incidence ratios are consistently reported compared to their prevalence values, indicating that - although the onset of new WMSDs is relatively limited - employees experience long-term chronic disorders, leading high prevalence numbers. This hypothesis is strengthened by the finding of Gold et al. [60] who states that employees affected by upper limb WMSDs were still experiencing the same disorder at least 1 year later.

More specific examples of chronic WMSDs were research by Leclerc et al. [52] who reported incidence ratios of $0.12,0.13$ and 0.06 for carpal tunnel syndrome, lateral epicondylitis and wrist tendinopathy, respectively. In accordance with Leclerc et al. [52], other included studies found similar incidence ratios of 0.14 and 0.12 for carpal tunnel syndrome, 0.02 for lateral epicondylitis and 0.03 for wrist tendinopathy [53, 54]. Notably, incidence values for these specific WMSDs tend to have higher consensus compared to other WMSDs. Although not similar in all variables, both Leclerc et al. [52] and Roquelaure et al. [54] investigated WMSDs during the same period (between 1996 and 1997) and worked with occupational physicians to obtain incidence data. In 
addition, carpal tunnel syndrome and lateral epicondylitis are two well-defined diagnoses compared to vague WMSD complaints in a nondelineated anatomical area. This clearly emphasizes the need for more precise definitions of WMSDs as well as standardized study procedures. In contrast to upper limb incidence data, there is a noticeable lack of information regarding neck WMSDs of industrial workers of Europe.

\section{Lower limb WMSDs}

Lower limb WMSDs were less prevalent than back or upper limb WMSDs with 29, 11, 33, 17\% obtained for the leg, hip, knee, ankle/feet WMSDs subgroups, respectively. EU-OSHA reported a similar trend with a 12month prevalence of 29 and 30\% lower limb WMSDs in 2010 and 2015, respectively [58]. It is possible that there are fewer risk factors present in an industrial setting for developing lower limb WMSDs compared to risk factors for upper limb WMSDs [2]. However, it is noteworthy that epidemiological research or risk assessments regarding lower limbs is generally under-represented which could mediate this discrepancy. Some contradicting trends in prevalence of WMSDs were found in lower limb subgroups regarding the examination method and the presence of biomechanical and/or psychosocial risk factors. Since prevalence values of WMSDs are not only influenced by abovementioned factors, it is plausible that the high heterogeneity, contributed to this absence of homogenous trends in included subgroups.

Three studies reported incidence rates of lower limb WMSDs, however overall incidence ratios could not be calculated due to the lack of sample size information [44-46]. Chen et al. [44] reported incidence ratios of 0.0121 (indicating that over the course of 1 year 1 out of 100 persons reported new lower limb WMSDs), 0.000049 and 0.0317 in the food, chemical and metallic industry, respectively. An incidence ratio of 0.000028 was obtained by cherry et al. [45] in the metallic industry. Substantially less information regarding lower limb incidence values is available in European reports and scientific literature to support this research. However, the trend of relatively low incidence values and higher prevalence values again indicate that recurrence of lower limb WMSDs is common among industrial workers. Insufficient time to heal or rehabilitation could contribute to these long-term disorders. Strategies that temporarily relieve the employee from hard labour and effective rehabilitation could benefit the healing process and limit recurrence of WMSDs.

\section{Limitations and future research}

It is evident that with the high obtained heterogeneity in the majority of subgroups, the included studies did not utilise uniformity in methodology and reporting strategies. Often, lack of information or discrepancies regarding demographic characteristics of included sample sizes, performed tasks, examining methods and examining periods challenged pooling and interpretation of obtained results. This resulted for the majority of subgroups in a limited number of included studies and considerable heterogeneity that could not be further investigated and challenges the interpretation of obtained results. In order to improve uniformity and generalisation of results, a criteria document for physical examination and standardized questionnaires (e.g. the NMQ) could be used. Further, assessment of risk of bias was performed with the tool as described by Hoy et al. [18] which was the most applicable for included studies. Kane and Shamliyan [61] previously argued that uniformity of this assessment tool is challenged by unclearly defined constructs. Therefore, guidelines for each item of the assessment tool were subjectively expanded and adjusted to this research's specific subject through discussion between authors. This self-interpreted variant of the initial definitions provided by Hoy et al. [18] could have influenced the risk assessment scores. Finally, only a limited number of studies reporting incidence values could be included due to scarcity in literature. This inhibited profound quantitative calculations of incidence ratios and highlights the need for more qualitative incidence research. Nevertheless, besides the high variability, the obtained high prevalence values, especially in food industries, indicate the need for effective rehabilitation and prevention strategies tailored to the unique characteristics of each industry type.

\section{Conclusion}

This review and meta-analysis aimed to provide an epidemiological overview of WMSDs in in twenty-first century Europe's secondary industries. Back (overall), shoulder/neck, neck, shoulder, lower back and wrist WMSDs were the most prevalent with mean values of 60\% (range 38-72\%), 54\% (range 18-83\%), 51\% (range $32-69 \%), 50 \%$ (range $23-78 \%$ ), 47\% (range 24-71\%) and 42 (range 14-64\%), respectively. Upper limb disorders were the most investigated WMSD in incidence studies and obtained incidence ratios from 0.04 to 0.26 . Data regarding lower limb and back WMSDs were scarce and incidence ratios could not be calculated. Although the onset of WMSDs in general appears to be limited, high prevalence values indicate long-term complaints. These results should be interpreted with caution due the high heterogeneity in the majority of subgroups. However, this highlights the need for future research in the epidemiology of WMSDs as well as the effectiveness of new prevention strategies. 


\section{Abbreviations}

WMSDs: Work-related Musculoskeletal Disorders; CDC: Centers for Disease Control and Prevention; EU-OSHA: European Agency for Safety \& Health at Work; NMQ: Nordic Musculoskeletal questionnaire

\section{Supplementary Information}

The online version contains supplementary material available at https://doi. org/10.1186/s12891-021-04615-9.

\section{Additional file 1.}

\section{Acknowledgements}

Not applicable.

\section{Authors' contributions}

All authors revised the manuscript critically for important intellectual content, approved the final version of the manuscript to be published, and agree to be accountable for all aspects of the work in ensuring that questions related to the accuracy or integrity of any part of the work are appropriately investigated and resolved. Renée Govaerts, Bruno Tassignon and Jo Ghillebert made substantial contributions to the conception or design and the work, the acquisition, analysis, and interpretation of data for the work, performed the literature search and also drafted and/or revised the work. Romain Meeussen, Bram Vanderborght and Kevin De Pauw made substantial contributions to the conception and design of the work, and interpretation of data. Ben Serrien, Toon Ampe, Sander De Bock and Ilias El Makrini made substantial contributions to the analysis, and interpretation of data for the work.

\section{Funding}

This work was supported by Horizon 2020; the SOPHIA-project (grant Agreement No. 871237).

\section{Availability of data and materials}

All data generated or analysed during this study are included in this manuscript.

\section{Declarations}

\section{Ethics approval and consent to participate}

Not applicable. The review and meta-analysis was developed and reported in accordance with the preferred reporting items for systematic reviews and meta-analyses (PRISMA).

\section{Consent for publication}

Not applicable.

\section{Competing interests}

The authors have no conflicts of interest to declare that are relevant to the content of this article.

\section{Author details}

${ }^{1}$ BruBotics, Vrije Universiteit Brussel, Brussels, Belgium. ${ }^{2}$ Human Physiology and Sports Physiotherapy Research Group, Vrije Universiteit Brussel, Brussels, Belgium. ${ }^{3}$ Strategic Research Program 'Exercise and the Brain in Health \& Disease: the added value of Human-centered Robotics', Vrije Universiteit Brussel, Brussels, Belgium. ${ }^{4}$ Sciensano Research Institute, Brussels, Belgium. ${ }^{5}$ Robotics research group, Vrije Universiteit Brussel and Flanders Make, Brussels, Belgium. ${ }^{6}$ Robotics research group, Vrije Universiteit Brussel and IMEC, Brussels, Belgium.

\section{Received: 14 May 2021 Accepted: 11 August 2021}

Published online: 31 August 2021

\section{References}

1. European Agency for Safety and Health at Work. Work-related MSDs: prevalence, costs and demographics in the EU. 2019.
2. da Costa BR, Vieira ER. Risk factors for work-related musculoskeletal disorders: a systematic review of recent longitudinal studies. Am J Ind Med. 2010;53(3):285-323. https://doi.org/10.1002/ajim.20750.

3. Santos J, Baptista J, Monteiro P, Miguel A, Santos R, Vaz M. The influence of task design on upper limb muscles fatigue during low-load repetitive work: A systematic review. Int J Industr Ergonom. 2016;52:78-91.

4. Roquelaure Y, Petit A, Fouquet B, Descatha A. Work-related musculoskeletal disorders: priority to prevention and coordination of the interventions. Rev Prat. 2014;64(3):350-7.

5. Bevan S. Economic impact of musculoskeletal disorders (MSDs) on work in Europe. Best Pract Res Clin Rheumatol. 2015;29(3):356-73. https://doi.org/1 0.1016/j.berh.2015.08.002

6. Szucs T, Nichol K. Economic and social impact of epidemic and pandemic influenza. Vaccine. 2006;24(44-46):6776-8.

7. Keech M, Scott AJ, Ryan PJ. The impact of influenza and influenza-like illness on productivity and healthcare resource utilization in a working population. Occup Med (Lond). 1998;48(2):85-90. https://doi.org/10.1093/occmed/48.2. 85.

8. de Carvalho MP, Schmidt LG, Soares MC. Musculoskeletal disorders and their influence on the quality of life of the dockworker: a cross-sectional study. Work. 2016;53(4):805-12. https://doi.org/10.3233/WOR-162249.

9. Roux CH, Guillemin F, Boini S, Longuetaud F, Arnault N, Hercberg S, et al. Impact of musculoskeletal disorders on quality of life: an inception cohort study. Ann Rheum Dis. 2005;64(4):606-11. https://doi.org/10.1136/ard.2004 020784.

10. European Commission. EUROPE 2020: A strategy for smart, sustainable and inclusive growth. 2010.

11. Rodriguez R, Warmerdam J, Triomphe C. The Lisbon Strategy 2000-2010: An analysis and evaluation of the methods used and results achieved; 2010.

12. de Looze MP, Bosch T, Krause F, Stadler KS, O'Sullivan LW. Exoskeletons for industrial application and their potential effects on physical work load. Ergonomics. 2016;59(5):671-81. https://doi.org/10.1080/00140139.2015.1081 988.

13. El Makrini I, Merckaert K, De Winter J, Lefeber D, Vanderborght B. Task allocation for improved ergonomics in Human-Robot Collaborative Assembly. Interact Stud. 2019;20:103-34.

14. Page MJ, McKenzie JE, Bossuyt PM, Boutron I, Hoffmann TC, Mulrow CD, et al. The PRISMA 2020 statement: an updated guideline for reporting systematic reviews. BMJ. 2021;372:n71.

15. Morgan RL, Whaley P, Thayer KA, Schünemann HJ. Identifying the PECO: a framework for formulating good questions to explore the association of environmental and other exposures with health outcomes. Environ Int. 2018;121(Pt 1):1027-31. https://doi.org/10.1016/j.envint.2018.07.015.

16. Centers for Disease Control and Prevention [Available from: https://www. cdc.gov/. Accessed 25 Apr 2021.

17. Ouzzani M, Hammady H, Fedorowicz Z, Elmagarmid A. Rayyan-a web and mobile app for systematic reviews. Syst Rev. 2016;5(1):210. https://doi.org/1 0.1186/s13643-016-0384-4.

18. Hoy D, Brooks P, Woolf A, Blyth F, March L, Bain C, et al. Assessing risk of bias in prevalence studies: modification of an existing tool and evidence of interrater agreement. J Clin Epidemiol. 2012;65(9):934-9. https://doi.org/10.1 016/j.jclinepi.2011.11.014

19. Higgins JP, Thompson SG. Quantifying heterogeneity in a meta-analysis. Stat Med. 2002;21(11):1539-58. https://doi.org/10.1002/sim.1186.

20. Aasmoe L, Bang B, Egeness C, Løchen ML. Musculoskeletal symptoms among seafood production workers in North Norway. Occup Med (Lond). 2008;58(1):64-70. https://doi.org/10.1093/occmed/kqm136.

21. Afonso L, Pinho ME, Arezes PM. In: Arezes PM, Baptista JS, Barroso MP, Carneiro P, Cordeiro P, Costa N, et al., editors. Prevalence of WMSD in the sewing sector of two companies of the footwear industry; 2014. p. 609-14.

22. Alexopoulos EC, Tanagra D, Konstantinou E, Burdorf A. Musculoskeletal disorders in shipyard industry: prevalence, health care use, and absenteeism. BMC Musculoskelet Disord. 2006;7(1):88. https://doi.org/10.1186/1471-2474-7-88.

23. Andersen $\mathrm{JH}$, Haahr JP, Frost P. Risk factors for more severe regional musculoskeletal symptoms: a two-year prospective study of a general working population. Arthritis Rheum. 2007;56(4):1355-64. https://doi.org/1 0.1002 /art.22513.

24. Bang BE, Aasmoe L, Aardal L, Andorsen GS, Bjørnbakk AK, Egeness C, et al. Feeling cold at work increases risk of symptoms from muscles, skin, and airways in seafood industry workers. Am J Ind Med. 2005:47(1):65-71. https://doi.org/10.1002/ajim.20109. 
25. Bonfiglioli R, Mattioli S, Spagnolo MR, Violante FS. Course of symptoms and median nerve conduction values in workers performing repetitive jobs at risk for carpal tunnel syndrome. Occup Med-Oxford. 2006;56(2):115-21. https://doi.org/10.1093/occmed/kqj007.

26. Claus M, Schuster M, Webendörfer S, Groneberg DA, Jähner J, Schiffmann D. Prevalence of back pain in employees of a German chemical company: Results of a large cross-sectional study. J Occup Med Toxicol. 2019;14(1):16.

27. de Zwart BC, Frings-Dresen MH, Kilbom A. Gender differences in upper extremity musculoskeletal complaints in the working population. Int Arch Occup Environ Health. 2001;74(1):21-30. https://doi.org/10.1007/s0042 00000188.

28. Descatha A, Leclerc A, Chastang JF, Roquelaure Y. Study grp repetitive W. medial epicondylitis in occupational settings: prevalence, incidence and associated risk factors. J Occup Environ Med. 2003;45(9):993-1001. https:// doi.org/10.1097/01.jom.0000085888.37273.d9.

29. Fouquet N, Bodin J, Descatha A, Petit A, Ramond A, Ha C, et al. Prevalence of thoracic spine pain in a surveillance network. Occup Med. 2015;65(2): 122-5. https://doi.org/10.1093/occmed/kqu151.

30. Harkness EF, Macfarlane GJ, Nahit ES, Silman AJ, McBeth J. Mechanical and psychosocial factors predict new onset shoulder pain: a prospective cohort study of newly employed workers. Occup Environ Med. 2003;60(11):850-7. https://doi.org/10.1136/oem.60.11.850.

31. Hussain T. Musculoskeletal symptoms among truck assembly workers. Occup Med (Lond). 2004;54(8):506-12. https://doi.org/10.1093/occmed/ kqh087.

32. Isolani L, Bonfigliolil R, Raffi GB, Violante FS. Different case definitions to describe the prevalence of occupational carpal tunnel syndrome in meat industry workers. Int Arch Occup Environ Health. 2002;75(4):229-34. https:// doi.org/10.1007/s00420-001-0304-2.

33. Kaergaard A, Andersen JH. Musculoskeletal disorders of the neck and shoulders in female sewing machine operators: prevalence, incidence, and prognosis. Occup Environ Med. 2000;57(8):528-34. https://doi.org/10.1136/ oem.57.8.528.

34. Le Manac'h AP, Ha C, Descatha A, Imbernon $E$, Roquelaure $Y$. Prevalence of knee bursitis in the workforce. Occup Med (Lond). 2012;62(8):658-60. https://doi.org/10.1093/occmed/kqs113.

35. Lima IAX, Moro ARP, Cotrim TP. Capacity index for work, psychosocial risk of work and musculoskeletal symptomatology in workers of a meat processing industry in Portugal. Adv Intelligent Syst Comput. 2019;820:289-95.

36. Nordander C, Ohlsson K, Balogh I, Hansson GA, Axmon A, Persson R, et al. Gender differences in workers with identical repetitive industrial tasks: exposure and musculoskeletal disorders. Int Arch Occup Environ Health. 2008;81(8):939-47. https://doi.org/10.1007/s00420-007-0286-9.

37. Olafsdóttir H, Rafnsson V. Musculoskeletal symptoms among women currently and formerly working in fish-filleting plants. Int J Occup Environ Health. 2000;6(1):44-9. https://doi.org/10.1179/oeh.2000.6.1.44.

38. Pope DP, Silman AJ, Cherry NM, Pritchard C, Macfarlane GJ. Association of occupational physical demands and psychosocial working environment with disabling shoulder pain. Ann Rheum Dis. 2001;60(9):852-8.

39. Roquelaure $Y$, Ha C, Leclerc A, Touranchet A, Sauteron M, Melchior M, et al. Epidemiologic surveillance of upper-extremity musculoskeletal disorders in the working population. Arthritis Care Res. 2006;55(5):765-78. https://doi. org/10.1002/art.22222.

40. Sormunen E, Remes J, Hassi J, Pienimaki T, Rintamaki H. Factors associated with self-estimated work ability and musculoskeletal symptoms among male and female Workers in Cooled Food-processing Facilities. Ind Health. 2009:47(3):271-82. https://doi.org/10.2486/indhealth.47.271.

41. Sundstrup E, Jakobsen MD, Jay K, Brandt M, Andersen LL. High intensity physical exercise and pain in the neck and upper limb among slaughterhouse workers: cross-sectional study. Biomed Res Int. 2014;2014: 218546.

42. Wixted F, Shevlin M, O'Sullivan LW. Distress and worry as mediators in the relationship between psychosocial risks and upper body musculoskeletal complaints in highly automated manufacturing. Ergonomics. 2018;61(8):1079-93. https://doi.org/10.1080/00140139.201 8.1449253.

43. Weyh C, Pilat C, Kruger K. Musculoskeletal disorders and level of physical activity in welders. Occup Med -Oxford. 2020;70(8):586-92. https://doi.org/1 0.1093 /occmed/kqaa169.
44. Chen Y, McDonald JC, Cherry NM. Incidence and suspected cause of workrelated musculoskeletal disorders, United Kingdom, 1996-2001. Occup Med (Lond). 2006;56(6):406-13. https://doi.org/10.1093/occmed/kql040.

45. Cherry NM, McDonald JC. The incidence of work-related disease reported by occupational physicians, 1996-2001. Occup Med. 2002;52(7):407-11. https://doi.org/10.1093/occmed/52.7.407.

46. Cherry NM, Meyer JD, Chen Y, Holt DL, McDonald JC. The reported incidence of work-related musculoskeletal disease in the UK: MOSS 1997-2000. Occup Med (Lond). 2001;51(7):450-5. https://doi.org/10.1093/occmed/51.7.450.

47. Cherry NM, Meyer JD, Holt DL, Chen Y, McDonald JC. Surveillance of workrelated diseases by occupational physicians in the UK: OPRA 1996-1999. Occup Med. 2000;50(7):496-503. https://doi.org/10.1093/occmed/50.7.496.

48. Descatha A, Roquelaure Y, Evanoff B, Mariel J, Leclerc A. Predictive factors for incident musculoskeletal disorders in an in-plant surveillance program. Ann Occup Hyg. 2007:51(3):337-44. https://doi.org/10.1093/annhyg/mel080.

49. Häkkänen M, Viikari-Juntura E, Martikainen R. Incidence of musculoskeletal disorders among newly employed manufacturing workers. Scand J Work Environ Health. 2001;27(6):381-7. https://doi.org/10.5271/sjweh.630.

50. Monaco MGL, Uccello R, Muoio M, Greco A, Spada S, Coggiola M, et al. Workrelated upper limb disorders and risk assessment among automobile manufacturing workers: a retrospective cohort analysis. Work. 2019;64(4):755-61.

51. Ha C, Roquelaure $Y$, Leclerc A, Touranchet A, Goldberg M, Imbernon E. The French musculoskeletal disorders surveillance program: pays de la Loire network. Occup Environ Med. 2009;66(7):471-9. https://doi.org/10.1136/ oem.2008.042812.

52. Leclerc A, Landre MF, Chastang JF, Niedhammer I, Roquelaure Y. Study grp repetitive W. upper-limb disorders in repetitive work. Scand J Work Environ Health. 2001;27(4):268-78. https://doi.org/10.5271/sjweh.614.

53. Ricco $M$, Signorelli $C$. Personal and occupational risk factors for carpal tunne syndrome in meat processing industry workers in northern Italy. Med $\mathrm{Pr}$. 2017;68(2):199-209. https://doi.org/10.13075/mp.5893.00605.

54. Roquelaure $Y$, Mariel J, Fanello S, Boissière JC, Chiron H, Dano C, et al. Active epidemiological surveillance of musculoskeletal disorders in a shoe factory. Occup Environ Med. 2002;59(7):452-8. https://doi.org/1 0.1136/oem.59.7.452.

55. Cumpston M, Li T, Page MJ, Chandler J, Welch VA, Higgins JP, et al. Updated guidance for trusted systematic reviews: a new edition of the Cochrane Handbook for Systematic Reviews of Interventions. Cochrane Database Syst Rev. 2019;10:Ed000142.

56. Briggs AM, Bragge P, Smith AJ, Govil D, Straker LM. Prevalence and associated factors for thoracic spine pain in the adult working population: a literature review. J Occup Health. 2009;51(3):177-92. https://doi.org/10.1539/joh.K8007.

57. Colombini D, Occhipinti E. Preventing upper limb work-related musculoskeletal disorders (UL-WMSDS): new approaches in job (re)design and current trends in standardization. Appl Ergon. 2006;37(4):441-50. https://doi.org/10.1016/j.apergo.2006.04.008.

58. Schneider E, Irastorza X, Copsey S. OSH in figures: work-related musculoskeletal disorders in the EU-facts and figures. Luxembourg: European Agency for Safety and Health at Work; 2010.

59. Buckle PW, Devereux JJ. The nature of work-related neck and upper limb musculoskeletal disorders. Appl Ergon. 2002;33(3):207-17. https://doi.org/1 0.1016/S0003-6870(02)00014-5.

60. Gold JE, d'Errico A, Katz JN, Gore R, Punnett L. Specific and non-specific upper extremity musculoskeletal disorder syndromes in automobile manufacturing workers. Am J Ind Med. 2009:52(2):124-32. https://doi.org/10.1002/ajim.20653.

61. Kane RL, Shamliyan T. Be specific and dare to generalize: do we need a rating form for every disease? J Clin Epidemiol. 2012;65(9):921-3. https://doi. org/10.1016/j.jclinepi.2012.03.003.

\section{Publisher's Note}

Springer Nature remains neutral with regard to jurisdictional claims in published maps and institutional affiliations. 